ARTICLE

\title{
Mutations in respiratory complex I promote antibiotic persistence through alterations in intracellular acidity and protein synthesis
}

\author{
Bram Van den Bergh (10 1,2,3, Hannah Schramke4, Joran Elie Michiels ${ }^{1,2}$, Tom E. P. Kimkes ${ }^{4}$, \\ Jakub Leszek Radzikowski ${ }^{4}$, Johannes Schimpf ${ }^{5}$, Silke R. Vedelaar ${ }^{4}$, Sabrina Burschel ${ }^{5}$, Liselot Dewachter ${ }^{1,2}$, \\ Nikola Lončar ${ }^{6}$, Alexander Schmidt (10 ${ }^{7}$, Tim Meijer ${ }^{4}$, Maarten Fauvart ${ }^{1,2,8}$, Thorsten Friedrich (1) ${ }^{5}$, \\ Jan Michiels (10) ${ }^{1,2,9 凶} \&$ Matthias Heinemann (i) $4,9 凶$
}

\begin{abstract}
Antibiotic persistence describes the presence of phenotypic variants within an isogenic bacterial population that are transiently tolerant to antibiotic treatment. Perturbations of metabolic homeostasis can promote antibiotic persistence, but the precise mechanisms are not well understood. Here, we use laboratory evolution, population-wide sequencing and biochemical characterizations to identify mutations in respiratory complex I and discover how they promote persistence in Escherichia coli. We show that persistence-inducing perturbations of metabolic homeostasis are associated with cytoplasmic acidification. Such cytoplasmic acidification is further strengthened by compromised proton pumping in the complex I mutants. While RpoS regulon activation induces persistence in the wild type, the aggravated cytoplasmic acidification in the complex I mutants leads to increased persistence via global shutdown of protein synthesis. Thus, we propose that cytoplasmic acidification, amplified by a compromised complex I, can act as a signaling hub for perturbed metabolic homeostasis in antibiotic persisters.
\end{abstract}

\footnotetext{
${ }^{1}$ Centre of Microbial and Plant Genetics, Department of Molecular and Microbial Systems, KU Leuven Leuven, Belgium. ${ }^{2}$ Center for Microbiology, Flanders Institute for Biotechnology, VIB, Leuven, Belgium. ${ }^{3}$ Department of Entomology, Cornell University, Ithaca, NY, USA. ${ }^{4}$ Molecular Systems Biology, Groningen Biomolecular Sciences and Biotechnology Institute, University of Groningen, Nijenborgh 4, Groningen, The Netherlands. ${ }^{5}$ Molecular Bioenergetics, Institute of Biochemistry, Albert-Ludwigs-University of Freiburg, Freiburg im Breisgau, Germany. ${ }^{6}$ Molecular Enzymology, Groningen Biomolecular Sciences and Biotechnology Institute, University of Groningen, Nijenborgh 4, Groningen, The Netherlands. ${ }^{7}$ Proteomics Core Facility, Biozentrum, University of Basel, Basel, Switzerland. ${ }^{8}$ imec, Leuven, Belgium. ${ }^{9}$ These authors jointly supervised this work: Jan Michiels, Matthias Heinemann.

凶email: jan.michiels@kuleuven.be; m.heinemann@rug.nl
} 
B acterial persistence describes the presence of phenotypic variants within an isogenic population that are transiently tolerant to otherwise lethal antibiotic therapy ${ }^{1-4}$. Such antibiotic-tolerant cells can endanger human health as they prolong therapy and can ultimately lead to relapsing infections ${ }^{1,3,5,6}$. In addition, tolerance levels are shown to increase during evolution under periodic antibiotic treatment ${ }^{7-10}$ and such increased tolerance levels were shown to proceed and accelerate the development of genetic resistance ${ }^{2,11-13}$.

Important advances towards understanding bacterial persistence have been made over the last years. First, toxin-antitoxin systems were found to be involved in persistence induction and awakening ${ }^{14-21}$. Second, the stringent response, a stress response that coordinates adaptation to stresses through altered levels of messenger nucleotides ppGpp and pppGpp (i.e. (p)ppGpp), has been linked to persistence via either increased levels of $(\mathrm{p})$ $\mathrm{ppGpp}^{22-24}$ or a plethora of pathways including the induction of the RpoS regulon ${ }^{25,26}$, the inhibition of major processes such as DNA replication, transcription, and translation ${ }^{27,28}$ and the activation of toxin-antitoxin systems ${ }^{18,29,30}$.

A recently growing body of evidence suggests that also the 'metabolic state' is a highly important and general signal in the context of persistence ${ }^{31,32}$. First, a variety of metabolic genes affect the frequency of persister cells ${ }^{33-37}$. Second, metabolic perturbations like diauxic ${ }^{28}$ and rapid nutrient shifts ${ }^{26,38}$ and entry in stationary phase $3,39,40$ induce persister cell formation, where certain rapid carbon shifts can force almost an entire population into an antibiotic-tolerant state in a metabolic fluxdependent manner ${ }^{38}$. Other indications for metabolic perturbations driving persistence include the observed decrease in ATP concentration ${ }^{15,21,41-43}$ as well as other imbalances of the metabolic network such as very fast growth ${ }^{44}$, enzyme saturation ${ }^{45}$, or a perturbed TCA cycle and/or respiration ${ }^{40,46,47}$. Given the multitude of various metabolic perturbations leading to persistence, we hypothesize that one generic signal exists that triggers persistence upon perturbed metabolic homeostasis. Yet, it is unknown what this trigger is and how it initiates persister cell formation.

Here, we describe a mechanism for how different perturbations of metabolic homeostasis lead to persister cell formation. Through laboratory evolution of persistence and whole-genome, population-wide sequencing, we identified respiratory complex I as a key mutational target for increased persister formation upon entry into stationary phase, in model and pathogenic E. coli strains. Complex I mutants with increased persistence display compromised proton translocation leading to significant cytoplasmic acidification upon perturbations of metabolic homeostasis. We propose that acidification induces the persister state in two ways: through activation of the RpoS regulon and, upon more severe cytoplasmic acidification, through shutdown of protein synthesis. Our findings show how cytoplasmic acidification, amplified by a compromised complex I, acts as a central signaling hub to connect a perturbed metabolic homeostasis with persister cell formation.

\section{Results}

Evolution towards increased persistence leads to mutations in nuo. To discover genetic determinants of persister cell formation upon perturbations of metabolic homeostasis, we exploited laboratory evolution selecting for increased persistence in an environment that cycles between dilution and regrowth to stationary phase and antibiotic treatments (Fig. 1a) ${ }^{8,10,48}$. In this work, we investigated the genomic changes that occurred in 37 populations of an E. coli lab strain that were previously evolved under such cycling environment with daily exposure to amikacin (ten populations), kanamycin, tobramycin, or gentamicin (nine populations each $)^{8}$. For comparison and generalization, we also included three populations of the uropathogen UTI89 that we evolved under daily exposure to amikacin in the current work, where we found 1000 to 10,000 -fold increases in persistence, similar to the previously evolved populations of the lab strain (Supplementary Fig. 1a).

To detect mutations in these $40 \mathrm{E}$. coli populations, we applied deep, whole-genome sequencing on population samples at the endpoint of evolution after 5-10 days. Initially focusing on mutations present at, or above, a frequency of 5\% (see "Methods"), we identified on average 3.15 mutations per population amounting to a total of 128 mutations across all populations (Supplementary Data 1; Supplementary Fig. 1b, c). Statistical analyses indicate that the evolved populations consist entirely of mutants (Supplementary Fig. 1d, e). Confirming the low mutational complexity as inferred from population-wide sequencing, genome sequences of randomly picked clones at endpoints only contained one or two mutations (Supplementary Table 1). Mutations are significantly enriched in coding regions of 29 genes, specifically targeting genes coding for inner-membrane proteins (Supplementary Fig. 1f-h). Several targets are repeatedly hit by identical mutations in different populations, and/or emerge as multiple alleles (Supplementary Fig. 1b, j-l).

Mutations are strongly enriched in the nuo operon, which is hit 41 times by 19 unique alleles (Fig. 1b, Supplementary Fig. 1b, $\mathrm{i}-\mathrm{k}$ ), with eight alleles reoccurring identically in as many as ten populations (Supplementary Data 1; Supplementary Fig. 11). When including mutations below our initial frequency cutoff of $5 \%, 237$ additional mutations were uncovered in the 29 previously identified target genes (Supplementary Data 2), showing a similar enrichment in the nuo operon (52 hits) with 28 newly identified minor alleles (Fig. 1b). As overall 38.3\% of each evolved population consists of nuo alleles (Supplementary Fig. 1m), we find that the nuo operon is the major target of evolution towards high persistence in our laboratory evolution experiment selecting for increased antibiotic survival upon perturbation of metabolic homeostasis through entry into stationary phase.

Mutations in nuo predominantly hit regions encoding transmembrane domains involved in proton translocation and are causal to high persistence. The E. coli nuo operon consists of 13 genes coding for $\mathrm{NADH} / \mathrm{ubiquinone}$ oxidoreductase (respiratory complex I). The complex serves as an entry point for electrons from NADH into the electron transport chain (ETC) (Fig. 2a) ${ }^{49}$. 87 out of the 93 identified mutations and 41 unique alleles are present in the genes nuoAHJKLMN of complex I. Notably, the structurally and functionally distinct part of the complex, encoded by nuoBCDEFGI, contains significantly fewer mutations (only six) (Supplementary Fig. 2a, b). NuoBCDEFGI forms the cytoplasmic arm that catalyzes NADH oxidation and that transports the electrons via a series of iron-sulfur clusters to the ubiquinone reduction site, $100 \AA$ apart ${ }^{50}$. NuoAHJKLMN builds the membrane arm that translocates protons across the membrane, thus contributing to the electrochemical membrane potential, i.e. proton motive force $(P M F)^{51,52}$ (Fig. 2a). The mutations we found are primarily in the proton translocating units of the membrane arm and specifically hit codons of amino acids enriched in hydrophobic residues (83 times) and of those residues located in transmembrane helices (64 times) (Fig. 2c, Supplementary Fig. 2a, b). While many of the altered amino acids are in close proximity to regions involved in proton translocation (Fig. 2a inset, Supplementary Fig. 2c, d) ${ }^{51-53}$, the mutations do not directly affect amino acids reported to be crucial for proton translocation, such as amino acids involved in long-range 


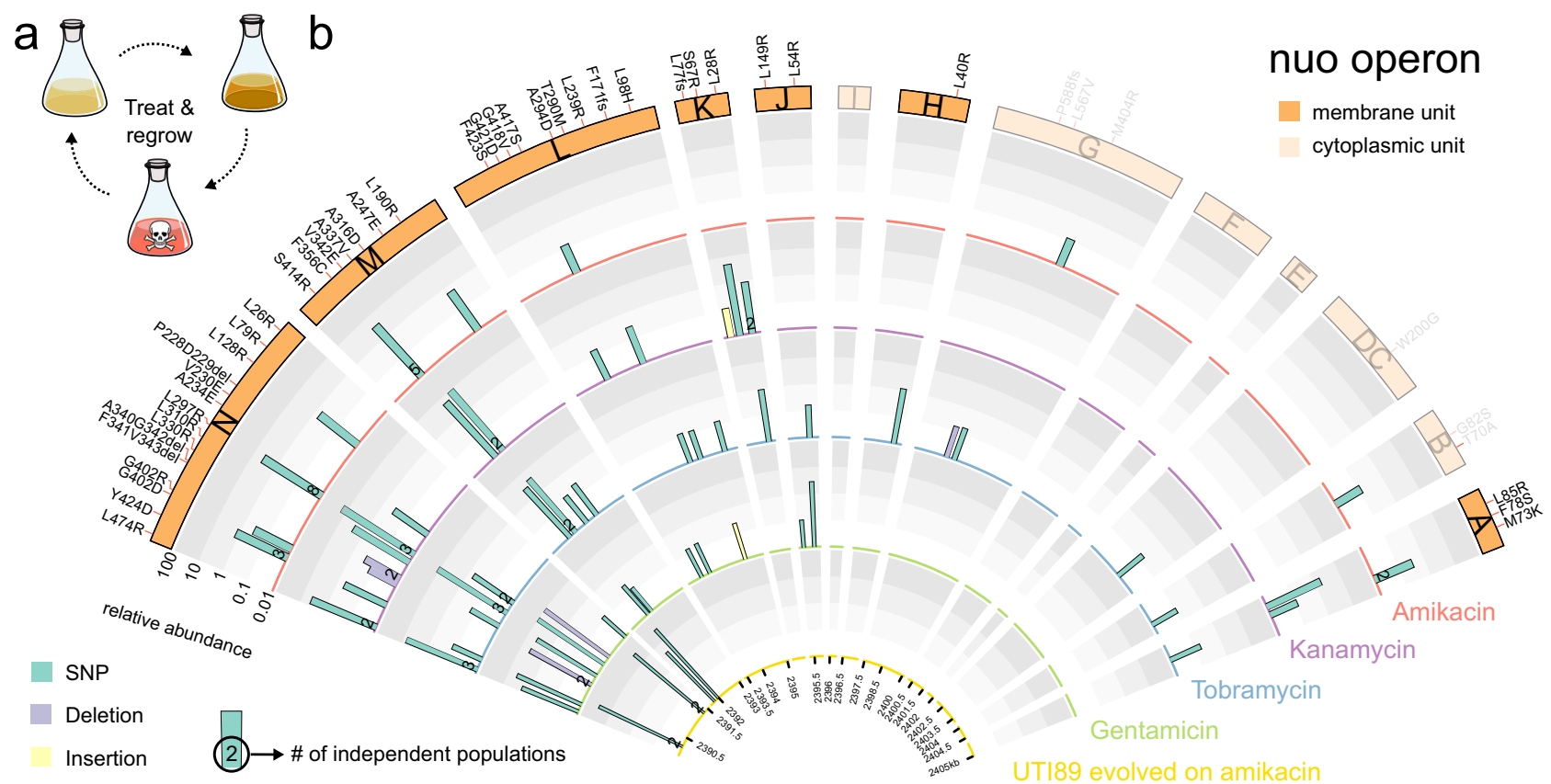

Fig. 1 As main target of evolution in E. coli towards increased persistence, the nuo operon is hit by mutations in genes primarily encoding membranespanning units. a Scheme of experimental evolution alternating daily between dilution and regrowth to stationary phase to a density of $1-5 \times 10^{9} \mathrm{CFU}$ ml ${ }^{-1}$ (respectively, light and dark orange) and antibiotic treatment (red), which selects for an increase in persistence. $\mathbf{b}$ A circos plot segment visualizes mutations that emerged in the evolved populations in genes of the nuo operon (orange rectangles with letters for each gene) which proved to be the main target of evolution (Supplementary Fig. 1). Genes encoding proteins that are part of the membrane-spanning arm of the complex and cytoplasmic domain are shown in bright and pale colors, respectively. Data of ten (amikacin), nine (kanamycin, tobramycin, and gentamicin) and three (UTI89) evolved populations were cumulated. Numbers in the bars show how many independently evolved populations contain that exact mutation $(\geq 2)$. The concentric arcs show the relative frequency of mutations in populations evolved on different antibiotics ( 4 outer arcs; all treated for $5 \mathrm{~h}$ with $400 \mu \mathrm{g} \mathrm{ml} \mathrm{m}^{-1}$ ) and in populations of the uropathogenic strain UTI89 evolved on amikacin (inner arc; for $5 \mathrm{~h}$ with $400 \mu \mathrm{g} \mathrm{ml}^{-1}$ ). Target regions are rescaled for visual representation and different types of mutation are shown in different colors. The outer ring shows the resulting amino acid change at the protein level for each of the mutations. See also Supplementary Fig. 1, Supplementary Tables 1-2 and Supplementary Data 1-2. Source data are provided as a Source Data file.

structural changes along a central axis in the membrane arm, or in outlining the proton half-channels, or in the actual binding and export of protons (Fig. 2a). Based on these findings, we expected that the mutations do not abolish the overall complex I function, but rather induce smaller functional changes in complex I, likely connected with proton translocation, leading to the observed increase in persistence.

To confirm causality of the mutations in nuo for persistence, we selected clones with single mutations in each one of the subunits L, M and $\mathrm{N}$ (called nuo* further on)-which are subunits that are all proposed to contain one distinct proton translocation path ${ }^{54}$-and restored the chromosomal wild-type alleles in these mutants by site-specific mutagenesis. Here we found that the strains again became susceptible towards amikacin, i.e. the antibiotic that imposed selective pressure during evolution (Fig. 2d). In addition, the minimal inhibitory concentration of amikacin remained unchanged in the $n u o^{*}$ mutants, excluding an increase in resistance as an explanation for their increased survival (Supplementary Fig. 2e). In fact, the $n u o^{*}$ mutants show clear cross-tolerance to other antibiotics in the stationary phase (Supplementary Fig. 2f-h), which is a known hallmark of persistence ${ }^{3}$. As already expected from the above-described characteristics of the mutations (e.g., that they are specific to the proton translocation arm and are not targeting crucial amino acids), the effect of the point mutations cannot simply be mimicked by destroying complex I functionality entirely, either by knocking out the individual subunits or the entire complex (Fig. 2e, Supplementary Fig. 2f, g). Restoring the wild-type alleles abrogates not only the amikacin tolerance as mentioned above, but also the multidrug tolerance of the point mutants for the related aminoglycoside kanamycin and for the unrelated fluoroquinolone ofloxacin (Supplementary Fig. 2i), demonstrating that $n u o^{*}$ mutations are causal to the high-persistence, multidrug-tolerant phenotype.

High-persistence-conferring variants of complex I oxidize NADH and transfer electrons but are impaired in proton translocation. To test whether the identified nuo* mutations indeed alter proton translocation, we produced and purified wildtype complex I and three variants, carrying a variation in either subunit $\mathrm{L}, \mathrm{M}$, or $\mathrm{N}$. Despite its huge size $( \pm 550 \mathrm{kDa})$ and its multitude of cofactors, we were able to purify wild-type complex I and its variants with retained activity from E. coli membranes through several chromatographic steps (Supplementary Fig. 3a, b; see "Methods"). Yields of the preparations were similar between the samples $\left( \pm 0.2 \mathrm{mg}\right.$ protein $\mathrm{g}^{-1}$ cells $)$ as were the banding patterns after SDS PAGE, both in line with previously obtained results ${ }^{55,56}$ (Supplementary Fig. 3c). Native gel electrophoresis confirmed stability and purity of the preparations, and in situ $\mathrm{NADH}$ oxidation demonstrated activity of the complexes (Supplementary Fig. 3d). The mutations did not introduce instability to the variants as judged from their "melting points"57 (Supplementary Fig. 3e, f). Thus, the purification yielded pure and active samples of the wild-type complex I and the three variants.

To profile the biochemical activities of the purified complexes, we made use 1) of proteoliposomes, i.e. vesicles made from E. coli polar lipids reconstituted with the individual preparations of complexes, of membrane fractions containing the wild-type complex I or the variants, and 2) of inside-out vesicles (ISOVs) 

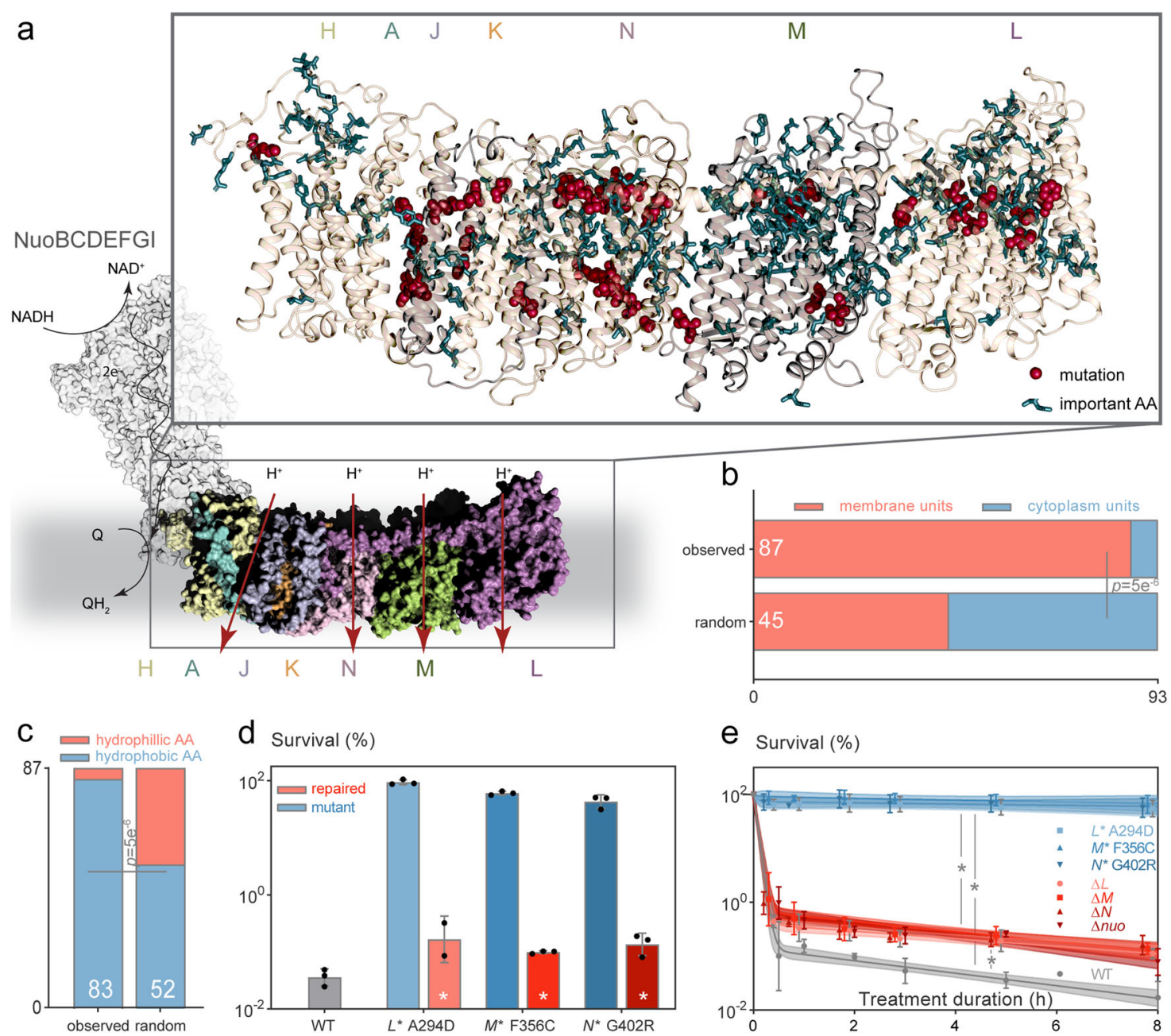

d Survival (\%)

e Survival (\%)
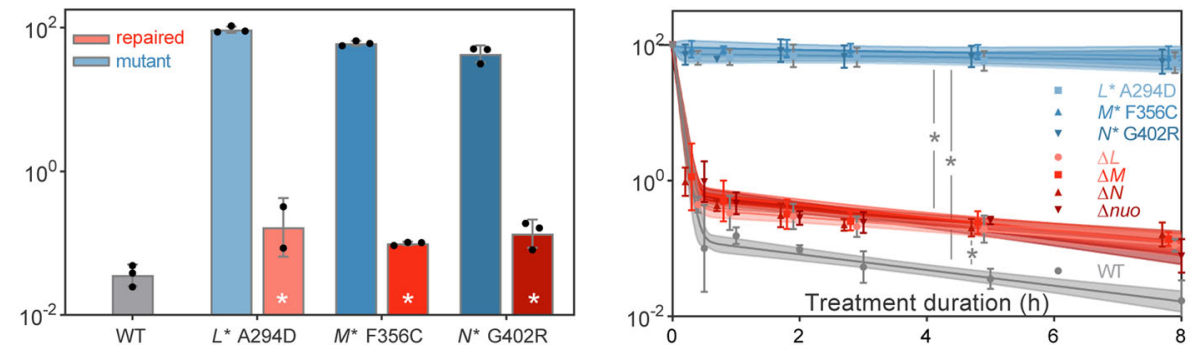

Fig. 2 High persistence-conferring nuo` mutations target hydrophobic amino acids in transmembrane subunits and do not cause a complete loss of function of the complex. a Modeled structure of E. coli complex I (see Methods). The cytoplasmic subunits NuoBCDEFGI (gray surfaces) catalyze NADH oxidation and transfer two electrons over a series of iron-sulfur clusters (not shown) to the quinone reduction site. The quinol flows further down the electron transport chain in the membrane (gray background) to be re-oxidized by terminal oxidases (not shown). The hydrophobic subunits NuoAHJKLMN (in colored surfaces) translocate four $\mathrm{H}^{+}$ions per molecule of $\mathrm{NADH}$ that is oxidized by the cytoplasmic subunits. The membrane part is magnified in the inset and is annotated with the positions of amino acid variants found in high-persistence mutants (red spheres) and with residues that are crucial for proton translocation (blue sticks, based on refs. ${ }^{51-53}$ ). b, c High-persistence mutations are significantly enriched in $\mathbf{b}$ the membrane units and $\mathbf{c}$ predominantly target hydrophobic amino acids ( $\mathrm{Chi}^{2}$ comparison to random mutations, see Methods). $\mathbf{d}$ The nuo mutations are causal for high persistence. Mutants with single mutations in each one of subunits $\mathrm{L}, \mathrm{M}$ and $\mathrm{N}$ lose their high tolerance for amikacin ( $5 \mathrm{~h}$ with $\left.400 \mu \mathrm{g} \mathrm{ml}{ }^{-1}\right)$ when nuo* mutations are repaired (mean \pm stdevs, $n=3 ;{ }^{*} p<0.0001$ for a within-strain comparison from a two-way ANOVA with Šidák's posttest). e Killing dynamics with amikacin $\left(400 \mu \mathrm{g} \mathrm{ml}^{-1}\right)$ confirm the high persistence of nuo* point mutants in stationary phase and show that their effect cannot be mimicked by a single gene or operon knockout (in red). A model describing biphasic killing dynamics (lines $\pm 95 \% \mathrm{Cls}$ ) was fitted to the data (means \pm stdevs, $n=3$; ${ }^{\star}$ fits are different based on AIC criterion). See also Supplementary Fig. 2. Source data are provided as a Source Data file.

prepared from E. coli membrane fractions (see "Methods"). Like proteoliposomes, the latter are closed compartments but additionally retain the full complexity of the in vivo membrane composition. NADH oxidation (and subsequent reduction of decyl ubiquinon in the membrane) was measured spectrophotometrically as a decrease in NADH absorption at $340 \mathrm{~nm}$, where we found that the liposomes reconstituted with wild-type complex I and the variants oxidized NADH at a similar rate (Fig. 3a and inset). Electrons from NADH ultimately flow down the ETC via the quinone/quinol couple to the terminal oxidases, where in well-aerated conditions oxygen is reduced to water. Respirometry on membranes that contain wild-type complex I or its variants as sole NADH dehydrogenase (see "Methods") revealed highly similar $\mathrm{O}_{2}$ reduction rates upon NADH addition (Fig. $3 \mathrm{~b}$ and inset). Thus, NADH oxidation and electron transfer are both not affected by the $n u o^{*}$ mutations.
To examine proton translation as third functionality of complex I, we made use of the fluorescence of 9-amino-6-chloro-2methoxyacridine (ACMA) that can be quenched upon alkalization induced by proton translocation of complex I, allowing to investigate differences in proton pumping (Supplementary Fig. 3g, h). Compared to wild-type complex I, all variants showed, to varying degrees (50-75\%), impaired proton translocation upon addition of NADH to the proteoliposomes (Fig. 3c). We confirmed this result by testing the activity of the variants in ISOVs (Fig. 3d). The ISOVs results furthermore quantitatively agreed with their more complex composition. As ISOVs also contain the terminal quinol oxidases, proton gradients measured with ACMA reflect the combined action of the quinol oxidases $\left(1 \mathrm{H}^{+} / \mathrm{e}^{-}\right.$for bd-type and $2 \mathrm{H}^{+} / \mathrm{e}^{-}$for bo3-type oxidase $)$and complex I $\left(2 \mathrm{H}^{+} / \mathrm{e}^{-}\right)^{58}$. ISOVs with complex I variants mainly translocate protons by the quinol oxidases and lack contribution 


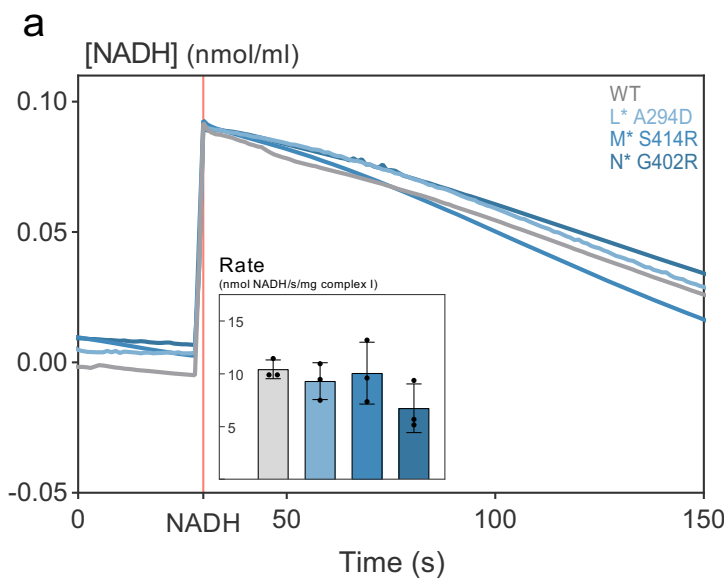

C

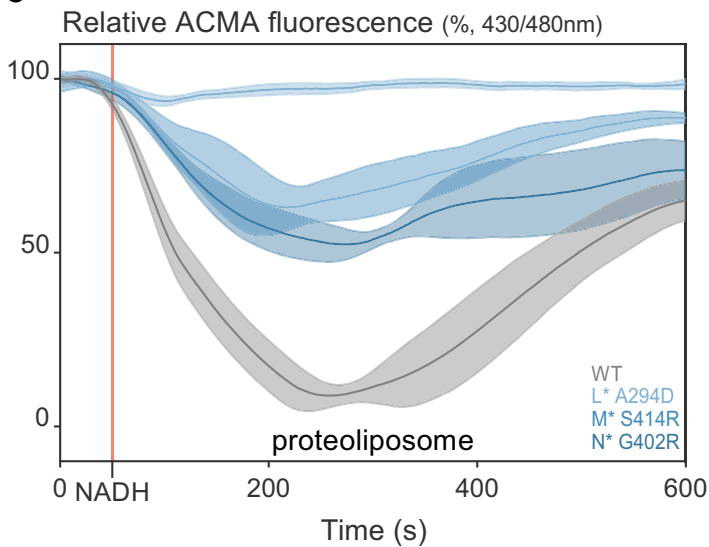

b

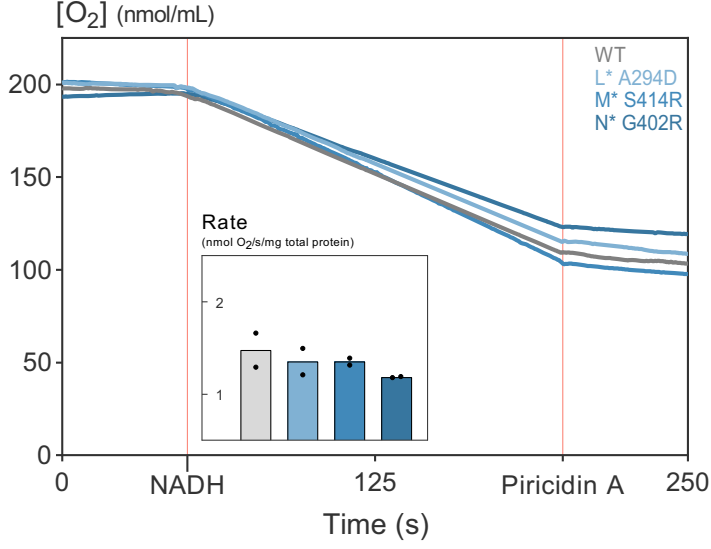

d

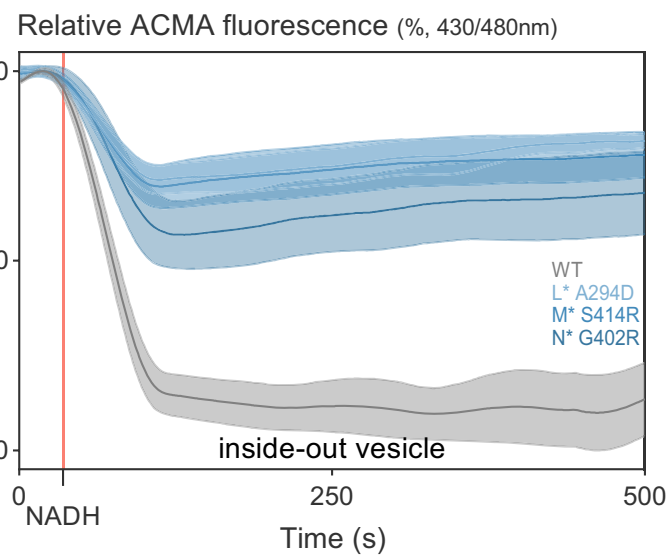

Fig. 3 High persistence-conferring nuo` variants are impaired in proton translocation. a Complex I variants show similar NADH/decyl ubiquinone oxidoreductase activity as the wild-type complex. Complexes were reconstituted in liposomes and NADH concentration was monitored at $340 \mathrm{~nm}$ (one representative of $n=3$ ). Average linear rates of NADH oxidation per $\mathrm{mg}$ complex I after NADH addition (inset, means \pm stdev, $n=3$ ) were not different (one-way ANOVA). Accounting for reconstitution efficiency, orientation, and activity of empty liposomes (see "Methods"), these rates amount to an overall NADH/decyl ubiquinone activity of $2.7 \mathrm{U} \mathrm{mg}^{-1}$, which is comparable to literature ${ }^{154}$. b The Nuo* variants and the wild-type complex transport electrons from NADH into the ETC with similar efficiency. Respirometry using a Clark-type electrode with membranes comprising complex I or its variants was used to measure the reduction of $\mathrm{O}_{2}$ by electrons which were released from $\mathrm{NADH}$ by complex I (one representative of $\mathrm{n}=2$ ). Average linear rates of $\mathrm{O}_{2}$ consumption per mg protein after $\mathrm{NADH}$ addition (inset, means) were not different (one-way ANOVA) and amount to an overall NADH oxidase activity of $0.16 \mathrm{U} \mathrm{mg}^{-1}$, which is comparable to literature ${ }^{154}$. The second red vertical line indicates addition of piericidin $\mathrm{A}$ (10 $\mu \mathrm{M}$ ), a potent complex I inhibitor, which indicates that $\mathrm{O}_{2}$ reduction indeed originates from $\mathrm{NADH}$ oxidation by complex I and that variants and wild-type complex I are equally sensitive to this inhibitor. $\mathbf{c}$, $\mathbf{d}$ Proton translocation is impaired in all variants as estimated from the difference in fluorescence quenching of the $\mathrm{pH}$-sensitive fluorophore ACMA in c proteoliposomes containing reconstituted complex I and in d ISOVs generated from membranes (means \pm sems; $n=3$ ). Relative fluorescent values were obtained by comparing the fluorescence to the start and through rescaling between 0 and $100 \%$ where $100 \%$ is the maximum value of each sample and $0 \%$ is the lowest value of the run (i.e. wild-type complex I which showed $\pm 50 \%$ quenching, similar to values found in literature ${ }^{154}$ ). Proteoliposomes are leaky and therefore revert the ACMA quench over time. ISOVs, while additionally showing the activity of the terminal oxidases, are much tighter and show no reversion. In $\mathbf{a}$, d, reactions were started by adding NADH (first red vertical line) and individual graphs are nudged horizontally and, in $\mathbf{a}$, b also vertically to allow comparison. See also Supplementary Fig. 3. Source data are provided as a Source Data file.

from complex I (so $1-2 \mathrm{H}^{+} / \mathrm{e}^{-}$), while complex I significantly contributes to proton translocation in wild-type ISOVs (so 3-4 $\mathrm{H}^{+} / \mathrm{e}^{-}$), which concurs with the 50-70\% lower ACMA quenching in ISOVs with complex I variants compared to wild-type ISOVs (Fig. 3d). Overall, these results demonstrate that the identified nuo* mutations in complex I target proton translocation and uncouple translocation from electron transfer.

Neither impaired antibiotic uptake nor decreased energy levels fully explains complex I-dependent increased persistence. Given their compromised proton translocation, we hypothesized that the nuo* mutants could have a decreased proton motive force (PMF). Previously, persisters were shown to tolerate aminoglycosides by means of a decreased PMF leading to lower antibiotic uptake $^{59}$. To test this hypothesis, we assessed the electrical gradient, i.e. the part of the PMF driving the uptake of charged aminoglycosides, using bis-(1,3-dibarbituric acid)-trimethine oxonol $\left(\mathrm{DiBAC}_{4}(3)\right)$, a fluorescent dye whose uptake inversely correlates with the electrical potential across the membrane ${ }^{18}$. Using flow cytometry, we assessed the level of $\operatorname{DiBAC}_{4}(3)$ of single cells to quantify their electrical gradient. Treatment with the dissipator carbonyl cyanide m-chlorophenyl hydrazone (CCCP) served as a depolarized control. The $n u o^{*}$ mutants showed a decreased electrical potential compared to the wild type (higher curves in Fig. 4a), resembling an expected consequence from the impaired proton translocation in complex I. However, the decrease in electrical gradient is minor-as expected, since the contribution of $\mathrm{pH}$ to the electrical gradient is usually only minor ${ }^{58,60}$ _and does not agree with the completely depolarized control that received CCCP 


\section{a}

$\%$ of cells with $\mathrm{DiBAC}_{4}(3)$ fluorescence higher than in $\mathrm{x}$ axis

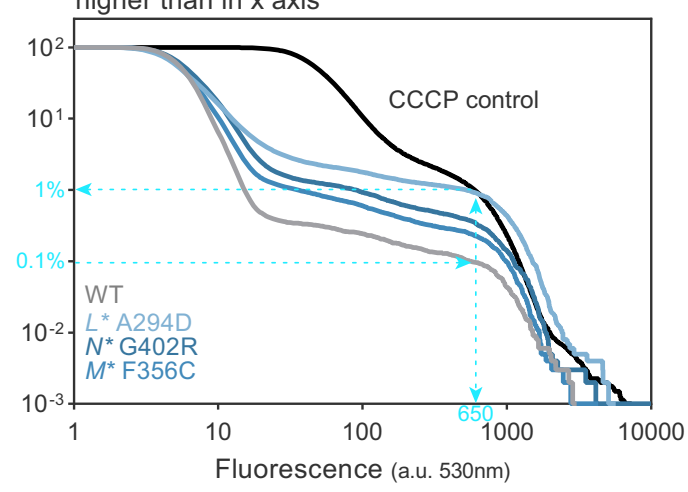

b

Intracellular amikacin ( $\mathrm{ng} / 10^{\wedge} 9$ cells)

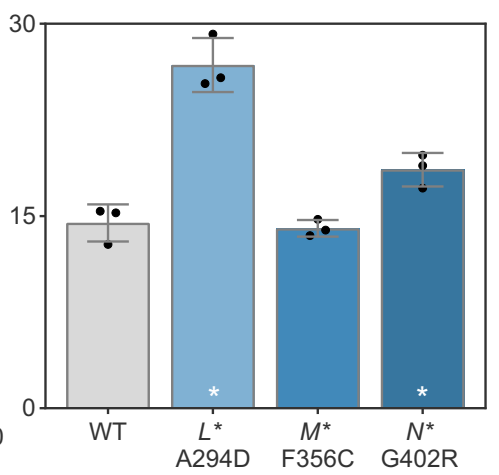

C

ATP:ADP (490/405 nm ratio)

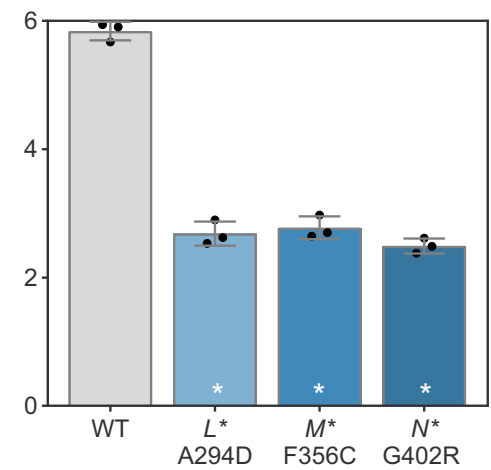

Fig. 4 High-persistence nuo ${ }^{\star}$ mutants have no impaired antibiotic uptake and do not solely depend on a decreased ATP:ADP ratio. a Single-cell

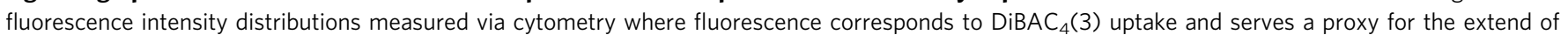

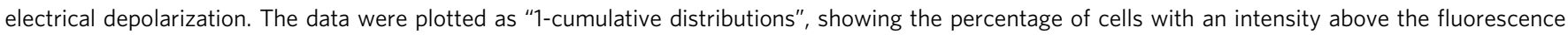
value specified in the $x$-axis to aid comparison between the strains. nuo* mutants in stationary phase show a minor decrease in electrical potential,

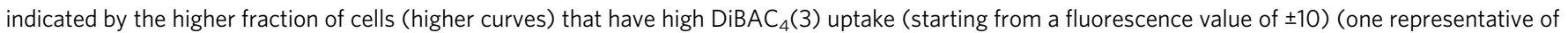

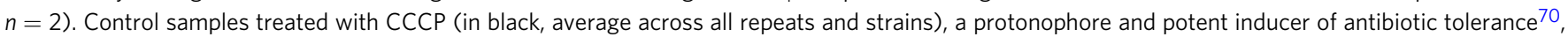
show the expected increase in dye uptake in all cells for a depolarized sample. Cyan lines, arrows and numbers were added to put the results in biological context: the wild-type persister level ( $0.1 \%$ of the population, see Fig. $2 \mathrm{e}$ ) corresponds to fluorescent level above 650 . Only < $1 \%$ of the nuo* mutants have such a high level of $\operatorname{DiBAC}_{4}(3)$, whereas these strains carry on average $50 \%$ persister cells (Fig. 2d, e, Supplementary Fig. $2 \mathrm{f}-\mathrm{i}$ ). b Intracellular amikacin concentrations of stationary phase cultures of nuo* mutants measured in a bioassay are similar to the wild type or higher, confirming that antibiotic uptake is not decreased in the high-persistence mutants (means \pm stdev, $n=3 ;{ }^{\star} p<0.01$ for comparison to the wild type from a one-way ANOVA with Dunnett's posttest). c In stationary phase (16-20 h after 1/100 dilution of an overnight culture), ATP:ADP ratios are significantly decreased as measured by the ratiometric GFP, Perceval (means \pm stdev, $n=3 ;{ }^{\star} p<0.0001$ for comparison to the wild type from a one-way ANOVA with Dunnett's posttest). However, nuo* mutants show similar decreases in ATP:ADP ratios in exponential phase when they do not display increased persistence (Supplementary Figs. $4 \mathrm{~b}, 2 \mathrm{~h}$ ). See also Supplementary Fig. 4. Source data are provided as a Source Data file.

treatment. More importantly, under the assumption that depolarization fully explains the tolerant state, the differences in electrical gradient do not match with the differences in persister levels. For example, the $0.1 \%$ persister level of the wild type agrees with an electrical potential that is only present in $1 \%$ or less of the cells in the nuo* mutants (cyan lines in Fig. 4a) while these mutants have a persister level of $\pm 50 \%$ (Fig. 2d, e, Supplementary Fig. 2f-i). Furthermore, and consistent with the small decrease in electrical gradient, nuo* mutants did not display decreased antibiotic uptake, as indicated by similar or even higher intracellular amikacin concentrations compared to the wild type (Fig. 4b). In addition, increased tolerance by a decreased antibiotic uptake as a result of a decreased electrical gradient would also not explain why the $n u o^{*}$ mutations also offer protection against ofloxacin (Supplementary Fig. $2 \mathrm{~g}$ ), a member of the fluoroquinolone antibiotics whose uptake does not dependent on an electrical gradient ${ }^{61-63}$ and which is also readily taken up by the $n u o^{*}$ mutants (Supplementary Fig. 4a). Altogether, these experiments show that a difference in antibiotic uptake does not explain the increased persister levels in the nuo* mutants.

Another consequence of the decreased proton translocation in the mutants could be a lowered ATP generation by the PMFdriven ATP synthase. Recently, a lower energy status has been linked to persistence ${ }^{15,21,41-43}$, while another study did not find decreased ATP levels in persisters ${ }^{26}$. We used a genetically encoded fluorescent reporter, Perceval ${ }^{64}$, to measure ATP:ADP ratios in stationary phase populations. The $n u o^{*}$ mutations led to a decreased ATP:ADP ratio compared to the wild type (Fig. 4c), which would be in line with the hypothesis of a de-energized cell status as cause of tolerance. However, the ATP:ADP ratios in the nuo* mutants are also decreased in the exponential phase (Supplementary Fig. 4e), a condition in which none of the mutants show increased tolerance (Supplementary Fig. 2h). While lower energy status might still contribute to the increased tolerance as an important prerequisite, we conclude that neither decreased antibiotic uptake nor a lower energy status by altered PMF can single-handedly explain the increased persister levels of the nuo* mutants, a result which we also confirmed for the evolved mutants of the uropathogenic strain (Supplementary Fig. 4c-g).

Increased cytoplasmic acidification in nuo* mutants is causal for increased persistence. Next, we hypothesized that the impaired proton translocation of the complex I variants might influence $\mathrm{pH}$ homeostasis during metabolic perturbations, which in turn could lead to increased persistence. To test this hypothesis, we used the ratiometric fluorescent $\mathrm{pH}$ sensor $\mathrm{pHluorin}{ }^{65}$ (Supplementary Fig. 4h) compared the cytoplasmic $\mathrm{pH}$ between the wild type and the high-persistence nuo* mutants. In exponential phase, a condition where we do not observe increased persistence in $n u o^{*}$ mutants (Supplementary Fig. $2 \mathrm{~h}$ ), the $\mathrm{pH}$ in the $n u o^{*}$ mutants did not significantly differ from the one in the wild type exhibiting an average $\mathrm{pH}$ value of $7.73 \pm 0.01$ (Supplementary Fig. 4j). However, in stationary phase, all highpersistence nuo* mutants showed a significantly more acidified cytoplasm compared to the wild type $\left(\mathrm{pH}_{\mathrm{i}}=6.79 \pm 0.04\right.$ versus $\mathrm{pH}_{\mathrm{i}}=7.39 \pm 0.05$; respectively, $p<0.0001$ ) (Fig. 5a, Supplementary Fig. 4i). The increased persistence in the nuo* mutants correlates with stronger acidification of the cytoplasm (Supplementary Fig. 4k), likely being elicited by the perturbation in metabolic homeostasis during entry into stationary phase.

To examine whether acidification of the cytoplasm is a general trait of metabolically induced persisters, we measured the cytoplasmic $\mathrm{pH}$ dynamically in time during well-controlled carbon nutrient shifts that are known to induce extremely highpersistence levels ${ }^{38}$. During exponential growth on glucose, we 


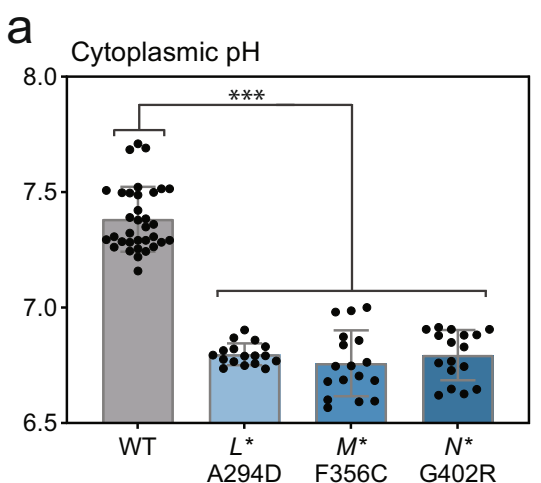

C

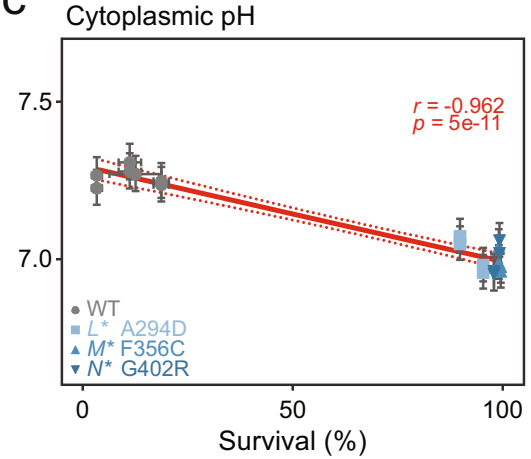

b

Cytoplasmic $\mathrm{pH}$

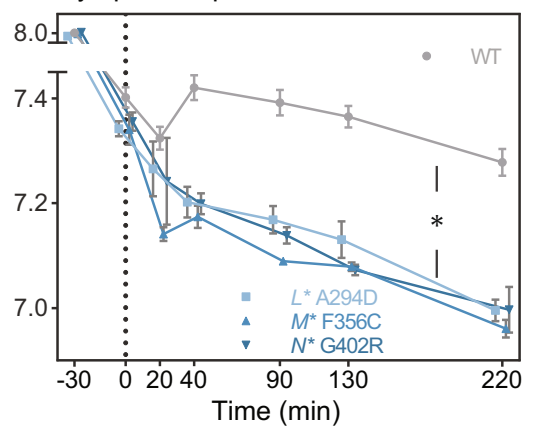

d

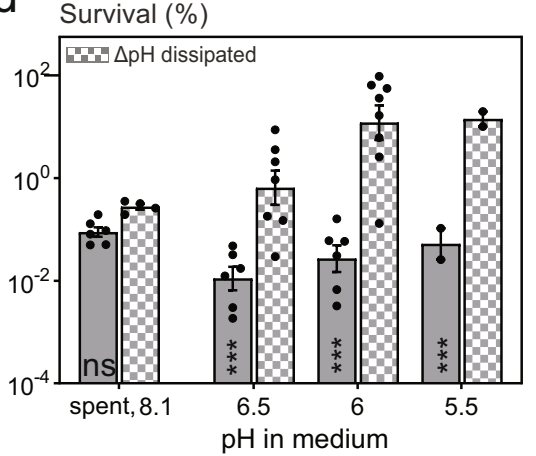

Fig. 5 Increased cytoplasmic acidification in nuo mutants is causal for increased persistence. a Cytoplasmic pH measured in cell populations using the ratiometric $\mathrm{pHluorin}$ sensor, is significantly decreased in the high-persistence nuo* mutants in comparison to the wild type in stationary phase, where the external $\mathrm{pH}$ is $8.1 \pm 0.06$ (means \pm stdevs, $\mathrm{n} \geq 17$; ${ }^{\star \star \star} p<0.001$ for a phenotype-level comparison from a linear mixed model). $\mathbf{b}$ Cytoplasmic $\mathrm{pH}$ of cells growing in exponential phase on $\mathrm{M} 9$ glucose medium decreases in all strains upon a shift to $\mathrm{M} 9$ fumarate medium (dotted line; $\mathrm{pH}=7$ ), $\mathrm{known}$ to induce persister formation. Dynamic measurement of cytoplasmic $\mathrm{pH}$ after a nutrient shift shows continued decreases over time only in the high-persistence nuo* mutants (means \pm sems, $n \geq 6$; ${ }^{*}$ slopes are different based on AIC criterion with the wild-type slope which is non-significantly different from 0 based on an $\mathrm{F}$ test). c Survival of amikacin treatment (for $4 \mathrm{~h}$ with $400 \mu \mathrm{g} \mathrm{ml}^{-1}$ ) assessed $30 \mathrm{~min}$ after the nutrient shift (in the same experiment as shown in $\mathbf{b}$ reveals a significant negative correlation between survival and cytoplasmic $\mathrm{pH}$ (means \pm sems, $\mathrm{n} \geq 4$ ). The linear regression line $( \pm 95 \%$ confidence interval) along with Pearson $r$ and $p$ values (top right corner) are shown in red. $\mathbf{d}$ Cytoplasmic acidification is causal for increased persister levels. Resuspending stationary phase cells in sterile spent medium buffered at different $\mathrm{pH}$ values increased the survival of amikacin treatment (for $5 \mathrm{~h} \mathrm{with} 400 \mu \mathrm{g} \mathrm{ml}{ }^{-1}$ ) from a pH of 6.5 and below in the wild type only when benzoate and methylamine are simultaneously added ( $\Delta \mathrm{pH}$ dissipators at $40 \mathrm{mM}$; boxed bars) to equilibrate cytoplasmic to extracellular $\mathrm{pH}$. Adding this weak acid-base pair does not influence tolerance levels in unbuffered spent medium that has a $\mathrm{pH}$ of $8.1 \pm 0.06$ (means \pm stdevs, $n \geq 2 ;{ }^{\star \star \star} p<0.001$ and $\mathrm{ns}=$ non-significant for within-pH comparison from a linear mixed model with Šidák's posttest). See also Supplementary Figs. 4-5. Source data are provided as a Source Data file.

again found a highly similar intracellular $\mathrm{pH}$ of $7.97 \pm 0.02$ in all strains (Fig. 5b), which dropped to $7.35 \pm 0.03$ directly after the shift to fumarate in all strains. While in the wild type, the $\mathrm{pH}$ stabilized around this level, the cytoplasm of the high-persistence nuo* mutants continued to acidify to $\mathrm{pH}$ values around 7.07 \pm 0.13 (at $220 \mathrm{~min}$ after the nutrient shift) (Fig. 5b). Thus, also with a completely different way of perturbing metabolic homeostasis and inducing persistence, we find that the cytoplasm acidifies, and that this acidification is stronger in the nuo* mutants. Remarkably, also here, the decreased $\mathrm{pH}$ of the $n u o^{*}$ mutants correlated with an increased tolerance (Fig. 5c, Supplementary Fig. 5a), generalizing the findings of a lowered $\mathrm{pH}$ and increased tolerance to a different perturbation of metabolic homeostasis.

Intrigued by the correlation between the decreased cytoplasmic $\mathrm{pH}$ and increased tolerance, both in the stationary phase and upon the sudden shifts between glucose and fumarate, we asked whether the increased cytoplasmic acidification in the nuo* mutants is causal for the increased tolerance levels. To test this, we aimed to lower the cytoplasmic $\mathrm{pH}$ in the wild type to see whether this also results in increased antibiotic tolerance. To accomplish this, we resuspended stationary-phase cells in spent medium buffered at a range of $\mathrm{pH}$ values and added a membrane- permeable pair of a weak acid and base, potassium benzoate and ethylamine hydrochloride ${ }^{65}$, to equilibrate the cytoplasmic $\mathrm{pH}$ with the $\mathrm{pH}$ of the spent medium. Here, using the wild type, we found that acidification of the cytoplasm increases persistence levels (Fig. 5d, Supplementary Fig. 5b). Thus, the decreased cytoplasmic $\mathrm{pH}$, which seems to be a general feature associated with persistence-inducing conditions involving metabolic perturbations, is further aggravated in the nuo* mutants, likely due to their compromised proton translocation, being causal for their increased tolerance.

RpoS contributes to increased persistence. Next, we asked how the lowered intracellular $\mathrm{pH}$ in the nuo* mutants could lead to increased tolerance. In our previous study, we found that the persisters generated through sudden nutrient shifts have an increased RpoS response with higher RpoS levels ${ }^{26}$. The stationary-phase specific nature of the increased persistence in the nuo* mutants also points to a role for RpoS, which is the 'stationary phase' sigma factor. As RpoS is posttranscriptionally and posttranslationally upregulated at low $\mathrm{pH}^{66-68}$, we hypothesized that the increased tolerance could be due to an increased $\mathrm{pH}$ induced RpoS response. 
a

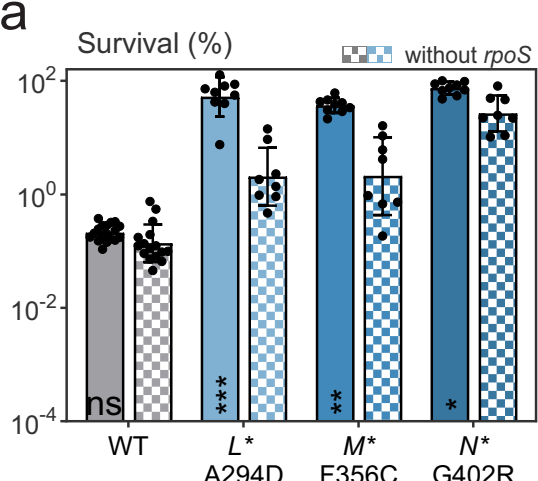

C PC1 (a.u.)

b
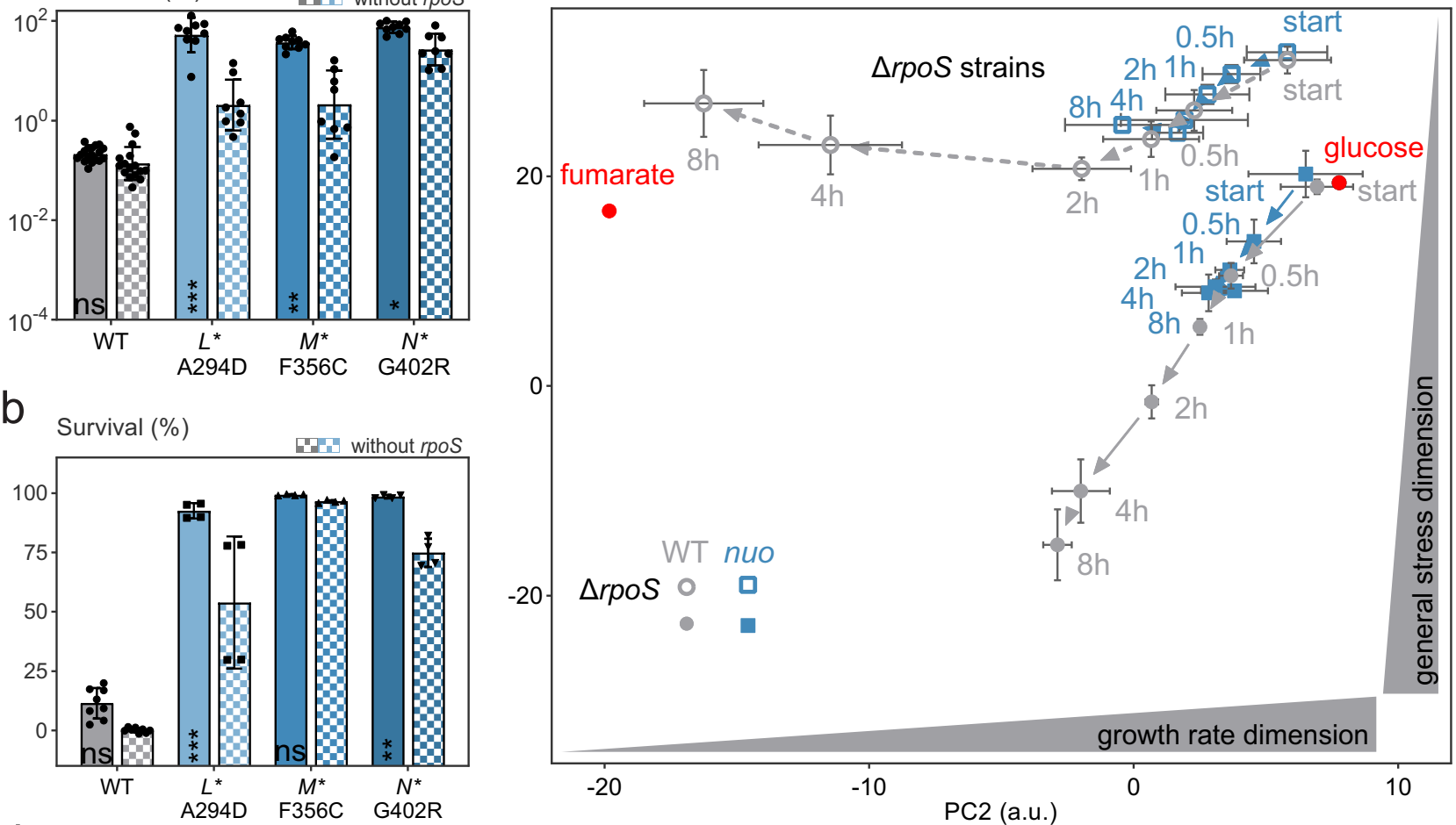

d

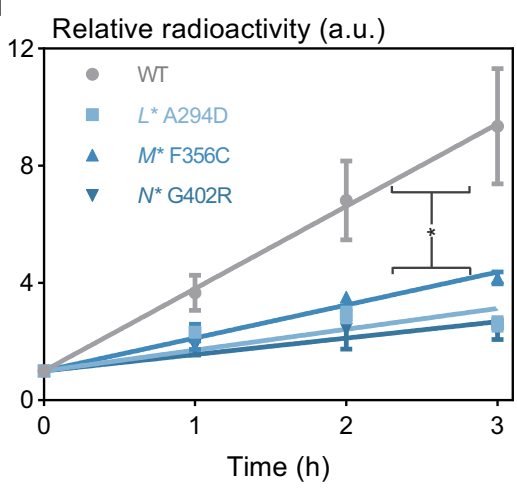

$\mathrm{e}$

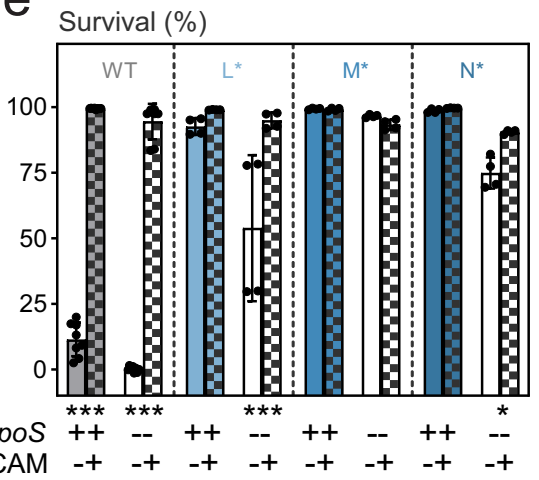

Fig. 6 RpoS and decreased protein synthesis contribute to increased persistence in nuo* mutants. $\mathbf{a}, \mathbf{b}$ Increased tolerance of the nuo mutants is partially RpoS-dependent in a stationary phase (amikacin for $5 \mathrm{~h}$ with $400 \mu \mathrm{g} \mathrm{ml} \mathrm{m}^{-1}$ ) and $\mathbf{b}$ upon the glucose-to-fumarate shift (amikacin for $4 \mathrm{~h}$ with $400 \mu \mathrm{g} \mathrm{ml}^{-1}$ ) (means \pm stdevs, $n \geq 4 ;{ }^{\star} p<0.05,{ }^{\star \star} p<0.01,{ }^{\star \star \star}{ }^{*} p<0.001$ and $\mathrm{ns}=$ non-significant for within-strain comparison from on a linear mixed model with Šidák's posttest). c Proteome changes after a shift from growth on glucose to fumarate (red dots) indicate that the proteome of high-persistence nuo mutants is in a locked state (means \pm stdevs, $n \geq 2$ ). The wild type with functional RpoS (closed gray symbols) shows an activation of the RpoS regulon as it moves down on the stress dimension of the PCA plot over time (see Methods), while removing rpoS (open gray symbols) leads to an adaptation to steadystate growth on fumarate and a migration along the growth rate dimension. Starting from $30 \mathrm{~min}$ after the shift to fumarate, the proteome of nuo* mutants remains unchanged, regardless of the presence of RpoS (in blue; average of nuo $M^{\star}$ and nuo $N^{\star}$ mutants). The nuoL mutant was omitted as, in this particular experiment, it did not show strong and persistent acidification and hence, displayed a deviating proteome change (Supplementary Fig. $5 f, g$ ), which further substantiates our finding. d Translation activity is lower in nuo* mutants in stationary phase as measured by the incorporation of radioactive $\mathrm{H}^{3}$-leucine (means \pm stdevs, $n \geq 2$; ${ }^{*} p<0.05$ for a phenotype-level comparison from a linear mixed model). e Chloramphenicol (CAM) supplementation ( $25 \mu \mathrm{g} \mathrm{ml}^{-1}$ at the time of the shift) induces tolerance towards amikacin (for $4 \mathrm{~h}$ with $400 \mu \mathrm{g} \mathrm{ml}{ }^{-1} ; 30 \mathrm{~min}$ after the shift) upon shifting from glucose to fumarate in the WT with/without $r p o S$ and the nuoL ${ }^{\star}$ and nuo $N^{\star}$ mutant lacking rpoS (means \pm stdevs, $n=4 ;{ }^{*} p<0.05$, ${ }^{\star \star \star} p<0.001$ for within-strain comparison between presence/absence of CAM from a linear mixed model with Šidák's posttest). See also Supplementary Figs. 5, 6. Source data are provided as a Source Data file.

Indeed, supporting a role for RpoS, we found that deleting rpoS reduces persistence of the $n u o^{*}$ mutants to some extent in stationary phase conditions (Fig. 6a) and also upon the glucoseto-fumarate shift (Fig. 6b). A mechanism that might explain how cytoplasmic acidification leads to increased RpoS levels is via increased ppGpp levels through a pH-based inhibition of both the spontaneous and the SpoT-dependent ppGpp hydrolysis, which we found to occur at $\mathrm{pH}$ values characteristic for persisters (Supplementary Fig. 6; see "Methods"). Surprisingly, however, high-persistence nuo* mutants lacking both RelA and SpoT, which are incapable of producing (p)ppGpp, remain highly antibiotic-tolerant in stationary phase conditions (Supplementary Fig. 5c). Thus, alternative and/or redundant routes need to exist that connect $\mathrm{pH}$ to $\mathrm{RpoS}$ induction. Moreover, since the nuo* 
mutants lacking RpoS are still more antibiotic tolerant than the wild type (Fig. 6a, b) and since the correlation between cytoplasmic $\mathrm{pH}$ (Supplementary Fig. 5d, e) and tolerance remains significant in those strains (Supplementary Figs. 4k, 1, 5a), a route additional to RpoS needs to connect the stronger decrease in cytoplasmic $\mathrm{pH}$ with the increased antibiotic tolerance.

Decreased $\mathrm{pH}$ inhibits translation and causes increased persistence. To investigate how the strong $\mathrm{pH}$ decrease in the nuo* mutants could cause the increased tolerance, we subjected the wild type and the $n u o^{*}$ mutant to proteome analyses at different time points during the controlled shift from glucose to fumarate, where we had found that the $n u o^{*}$ mutants acidify more strongly than the wild type (Fig. 5b). We mapped the high-dimensional proteome data onto a two-dimensional space created by a previously performed principal component analysis of a similar dataset (Fig. 6c, Supplementary Fig. 5f; see "Methods") 26,69. One dimension reflects a "growth axis" along which proteomes from cultures growing at steady-state in different growth conditions cluster. The other dimension reflects a "stress axis". It was previously found that the proteome of the wild type after the nutrient shift leading to persister formation, moved down the "stress" dimension, in a process that continued over $>8 \mathrm{~h}^{26}$.

Here, agreeing with these previous results, we find that wildtype cells, when shifted from glucose to fumarate, display proteome dynamics moving down the stress dimension (closed, gray symbols in Fig. 6c), characterized by induction of the global stress response ${ }^{26}$. Secondly, we found that the proteome of the wild-type strain lacking rpoS (open, gray symbols in Fig. 6c) changes steadily over time towards a proteome of cells with normal steady-state growth on fumarate (red dot in Fig. 6c). This is in line with the previous finding that, in an $\Delta r p o S$ mutant, almost all cells resume growth after a rapid shift to fumarate ${ }^{26}$. Furthermore, this observation supports our earlier conjectured hypothesis $^{32}$ that an $\Delta r p o S$ mutant strain invests resources in restoring metabolic homeostasis, and not in massive activation of stress responses, which is also corroborated by a somewhat restored $\mathrm{pH}$ homeostasis (i.e., reversal of the initial acidification) in the absence of RpoS in the wild type after a shift to fumarate (Supplementary Fig. 5d).

The proteome of $n u o^{*}$ mutants carrying a functional rpoS gene (closed, blue symbols in Fig. 6c) showed changes in the direction of an induced stress response for the first $30 \mathrm{~min}$ after the nutrient shift, similar to the changes of the wild-type proteome. Surprisingly, however, after $30 \mathrm{~min}$ the proteome stops changing abruptly (Fig. 6c), suggesting that the proteome might be locked in a frozen state with no further net turnover of proteins (i.e., either no or a balanced translation and degradation). Notably, it was also around $30 \mathrm{~min}$ that the cytoplasmic $\mathrm{pH}$ started to deviate between the wild type and the nuo* mutants (Fig. 5b) and that a correlation emerged between cytoplasmic $\mathrm{pH}$ and antibiotic tolerance (Fig. 5c, Supplementary Fig. 5a). Additionally, nuo* mutants lacking rpoS show a similar halt in proteome dynamics (Fig. 6c), which is in agreement with the only minor decreases in tolerance upon deletion of rpoS in these backgrounds. Thus, these data suggest that after about $30 \mathrm{~min}$, which is when the $\mathrm{pH}$ in the nuo* mutants has dropped to $\mathrm{pH}$ values below 7.2 , net protein turnover comes to a halt.

To test whether protein turnover is also halted in the stationary phase conditions on complex medium, we performed radioactive labeling experiments to follow protein synthesis. Here, we found that translation is indeed impaired in all $n u o^{*}$ mutants under the conditions that they were originally selected for (Fig. 6d), excluding the possibility that any net changes in the proteome were masked by a translation activity that balances out protein degradation. Thus, we conclude that the strong cytoplasmic acidification in the nuo* mutants halts changes in proteome turnover. We conjectured that halted protein translation, because of inactivity of amikacin's antibiotic targets, could render the nuo* mutants more tolerant than the wild type.

To test whether the halted translation in the $n u o^{*}$ mutants is indeed responsible for increased persistence, we artificially blocked translation after shifting cells to fumarate by the addition of a sublethal dose of chloramphenicol and checked for tolerance against amikacin after $30 \mathrm{~min}$. Here, we found that such a pretreatment, similar to what was found previously ${ }^{70}$, results in a strong induction of persistence in the wild type, comparable to the levels in the nuo* mutants without pretreatment (Fig. 6e). Additionally, such an artificial inhibition of translation also abolishes the need for rpoS both in the wild type and $n u o^{*}$ mutants. Thus, we conclude that the $\mathrm{pH}$-induced halted translation in the nuo* mutants is indeed the main factor responsible for the observed increased persistence against amikacin, bypassing the need for a full-blown activation of stress responses by RpoS as mechanism for tolerance as is the case for the wild type.

\section{Discussion}

Here, we present a mechanistic model, which explains how persister cells are formed upon perturbations of metabolic homeostasis (Fig. 7). In this model, strong metabolic perturbations, such as the shift into stationary phase or abrupt nutrient shifts, lead to cytoplasmic acidification, likely through protons released through continued ATP consumption without ATP regeneration, which has been linked to acidification in other systems ${ }^{71-75}$. Mutations in complex I that compromise proton translocation result in a further decreased cytoplasmic $\mathrm{pH}$ upon metabolic perturbations. Part of the following induction of persistence, especially in the wild type, is conferred by the RpoSdependent global stress response. In the $n u o^{*}$ mutants with increased cytoplasmic acidification, persistence is additionally conferred by a global halting of protein synthesis. At the system level, additional feedback and interaction mechanisms exist: an RpoS response inhibits restoration of metabolic homeostasis ${ }^{26}$ and the $\mathrm{pH}$-induced halted protein synthesis further prevents restoration of homeostasis, while also halting a further activation of the RpoS response. Our newly identified mechanism also agrees with a growing body of recent research that suggest that bacterial metabolism, chemiosmotic homeostasis and membrane bioenergetics - in addition to drug uptake and activity of primary targets-dictates the lethality of bactericidal antibiotics ${ }^{76-78}$.

Our work identifies complex I as an important persistence factor. Loss-of-function mutations in complex I (e.g., transposon or frameshift mutations) are long known to abrogate the growth advantage in long-term stationary-phase conditions of rpoS 'GASP' mutants in absence of antibiotics ${ }^{79}$. More recently, such mutations were shown to increase gentamicin tolerance in $E$. coli $i^{80}$ and aminoglycoside resistance in E. coli ${ }^{81-83}$ and Pseudomonas aeruginos $a^{84}$ through a reduced PMF and antibiotic uptake. Such an effect could also be mimicked by artificially decreasing complex I activity $85-88$ and was recently proposed to underly the antibiotic tolerance of a point mutant in $n u o N$ in $P$. aeruginos $a^{89}$. Here, we describe a new way in which complex I alters antibiotic susceptibility, i.e. by changes that solely impair proton translocation activity of the complex. Mutations identified in this work specifically lie in the proximity of the three previously confirmed proton pathways in NuoL, $\mathrm{M}$ and $\mathrm{N}$ and were found at the interface of the NuoAHJK subunits, thereby corroborating a previously hypothesized fourth channel at this site $^{51,53,90}$. Additionally, the $n u o^{*}$ mutants still take up antibiotics 


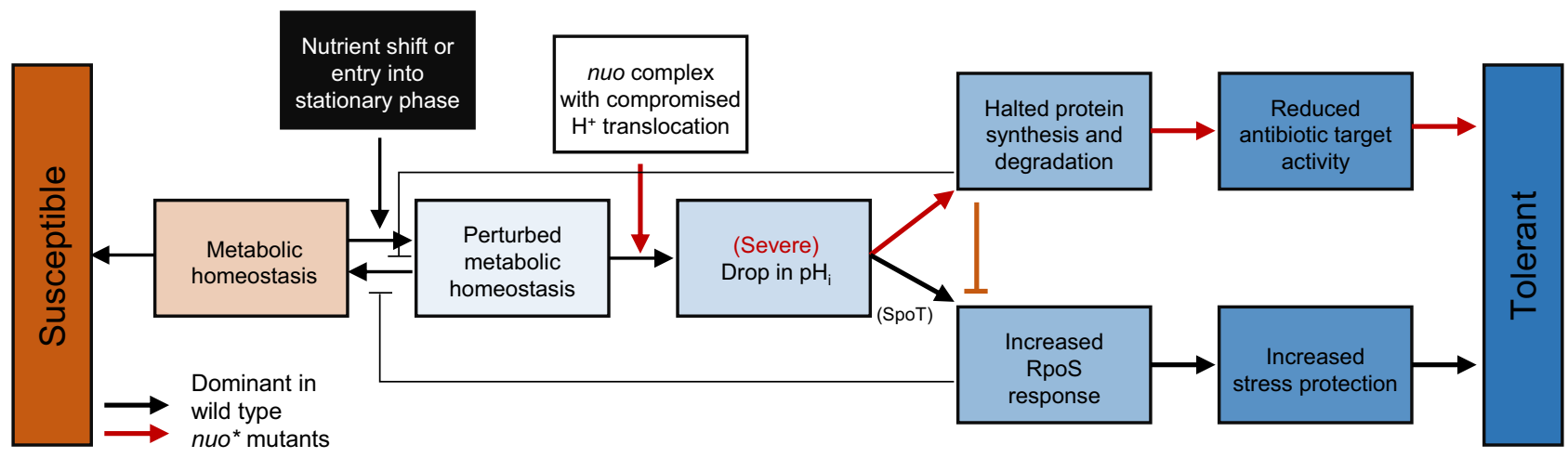

Fig. 7 Model connecting metabolic homeostasis, cytoplasmic acidification and two paths for persister cell formation. A strong perturbation of metabolic homeostasis by nutrient shifts or entry in stationary phase acidifies the cytoplasm. Such acidification could occur due to an imbalance between ATP consumption and regeneration. In these conditions, complex I acts as a regulator of cytoplasmic pH: wild-type proton translocation activity counterbalances acidification; impaired proton translocation in the nuo* mutants enhances cytoplasmic acidification after \pm 30 min of perturbation of metabolic homeostasis. Mild cytoplasmic acidification leads to increased persistence by an increased RpoS response, possibly mediated by inhibition of SpoTmediated hydrolysis of (p)ppGpp (dominant path in black arrows in the wild type). Stronger cytoplasmic acidification halts protein synthesis and renders antibiotic targets inactive leading to a second persistence mechanism (dominant path in red arrows in nuo* mutants with impaired complex I function.) At various points in this model, feedback loops are in place that affect persister formation. For instance, RpoS inhibits adaptation to maintain metabolic homeostasis, recovering from the initial pH drop. In turn, strong intracellular acidification via halted protein synthesis inhibits full RpoS activation.

and show cross-tolerance towards other antibiotics, in contrast to the collateral hypersensitivity towards other antibiotics of (lossof-function) mutations in nuo (and other genes) described in the context of aminoglycoside resistance due to reduced drug uptake $^{83,91}$. Therefore, the nuo* mutations identified in the current study constitute a new mechanism in which complex I influences antibiotic survival in bacteria, i.e. by inducing persister formation through increased cytoplasmic acidification upon strong perturbations of metabolic homeostasis.

In this work, we uncovered that cytoplasmic acidificationinduced by perturbations of metabolic homeostasis, i.e. upon nutrient shifts or entry into stationary phase-acts as a signal for persister formation in E. coli. In fact, a drop in cytoplasmic $\mathrm{pH}$ was observed in Salmonella upon macrophage engulfment and this acidification was furthermore important for the injection of modulating effector proteins that allow Salmonella to survive inside the vacuole ${ }^{92}$. At the same time, but without a connection to cytoplasmic acidification, Helaine and colleagues showed that macrophage internalization induced intracellular persister formation $^{93}$. Indole, the inter-kingdom signal molecule that was previously connected to the persister state ${ }^{94-96}$, was shown to regulate intracellular acidification upon entry in stationary phase $^{97}$ although very recently, the tryptophanase that catalyzes the formation of indole, not indole itself, was found to cause a lower $\mathrm{pH}$ in persisters ${ }^{98}$. In Mycobacterium smegmatis, bactericidal activity of antibiotics was linked to cytoplasmic alkalization during treatment ${ }^{99}$ while cytoplasmic acidification was recently found important for fully fledged resistance in marR $E$. coli mutants ${ }^{100}$. Beyond persisters and antibiotic survival, a drop in the cellular $\mathrm{pH}$ is more commonly associated with metabolic perturbations and dormancy. For instance, the $\mathrm{pH}$ of spores of Bacillus megaterium and Bacillus cereus is 1 to $1.3 \mathrm{pH}$ units lower than the $\mathrm{pH}$ of growing cells, but rises rapidly upon germination ${ }^{101-103}$. In yeast, cytoplasmic $\mathrm{pH}$ was identified as a second messenger for glucose levels where low intracellular glucose induces acidification ${ }^{104}$. Even beyond microbial life, a $\mathrm{pH}$ drop seems to be a dormancy signal, as intracellular $\mathrm{pH}$ is a dormancy marker in strawberry plants ${ }^{105}$, and has been shown to regulate dormancy in shrimp embryos ${ }^{106}$. Thus, across different species and even kingdoms, cytoplasmic acidification might act as a 'low-level' signal, indicating strong perturbations of metabolic homeostasis, ultimately causing cells to enter 'safe states' such as dormancy or persistence.

Since RpoS is known to be upregulated at low $\mathrm{pH}^{66-68}$ its role in persistence after cytoplasmic acidification was somewhat expected. How cytoplasmic acidification influences the here uncovered halting of protein turnover as they occur in the nuo* mutants after $30 \mathrm{~min}$ (Fig. 5d), is less clear as mechanisms to downregulate protein synthesis during growth arrest are not fully understood $^{107}$. The sudden and simultaneous stop of both protein production and degradation, as we inferred from both the sudden stop in proteome changes and the decreased translation activity (Fig. 6c, d), could result from cytoplasmic acidification leading to-as shown in yeast-a glass-like state with decreased intracellular dynamics that induces dormancy ${ }^{75}$, similar to the glassy cytoplasm connected with dormancy in other species ${ }^{108-111}$. Additional evidence for the significance of cytoplasm fluidity is the recently identified correlation of protein aggregate formation with persistence ${ }^{41,112-115}$, while, at the same time, widespread and $\mathrm{pH}$-dependent aggregation of native-like proteins underlies the transition of the cytoplasm to a solid-like state $^{75}$. Recently, mutations in translation-related genes were selected during laboratory evolution towards increased persistence and shown to affect translation more specifically ${ }^{116}$. Similarly, changes in cytoplasmic $\mathrm{pH}$ could also modulate ribosome activity more directly than via the induction of a global glass-like dormant cytoplasm, for example by unbalancing the ionic fluxes of other cations like $\mathrm{K}^{+}, \mathrm{Ca}^{2+}$, and $\mathrm{Mg}^{2}$, that were recently associated with ribosome functioning and/or antibiotic killing $76,78,117$. Therefore, a decrease in cytoplasmic $\mathrm{pH}$ could lead via a myriad of ways (e.g., cytoplasmic rigidity, ionic imbalances) to halted protein turnover and thus to increased tolerance.

With this work, we have unraveled the molecular basis of how metabolically induced persister cells are formed, where the dynamic nature of cytoplasmic $\mathrm{pH}$, as a signal for strong metabolic perturbations, resembles a global connector of metabolism with persister formation. The generic nature of this mechanism, converging at cytoplasmic acidification, is consistent with the fact that numerous effects, including perturbations of many metabolic genes ${ }^{118,119}$, abrupt nutrient shifts, changes in extracellular $\mathrm{pH}^{93}$, 
sublethal concentrations of antibiotics and oxidative stress ${ }^{120}$ have all been observed to induce tolerance and could all converge in intracellular acidification.

\section{Methods}

Bacterial strains, media, and cultivation. All bacterial strains used in this study are listed in Supplementary Data 3, with the most important strains being SX43 or SX2513 (the ancestral lab strains used in most of our experiments as "wild type" or WT, which are close relatives to BW25113), and UTI89 (a uropathogenic E. coli strain). Genomic mutants were constructed with either P1vir phage transduction as described before ${ }^{121}$ or through homologous recombineering described before ${ }^{122}$. Where possible, mutants from an ordered gene knockout library in E. coli, the Keio collection ${ }^{123}$ were used as template to create new mutants and they were cured from their $\mathrm{Km}^{\mathrm{R}}$-cassette by transformation with pCP20 expressing the FLP recombinase. At all steps, validity of mutants was checked by either PCR with gel electrophoresis or targeted Sanger sequencing or both using primers listed in Supplementary Data 4.

Experiments were performed using M9 minimal medium prepared as previously described ${ }^{38}$, Mueller Hinton Broth (MHB, Becton Dickinson; widely used in antibiotic sensitivity testing), lysogeny broth (LB) medium $\left(10 \mathrm{~g} \mathrm{~L}^{-1} \mathrm{NaCl}\right.$, $10 \mathrm{~g} \mathrm{~L}^{-1}$ trypton, $5 \mathrm{~g} \mathrm{~L}^{-1}$ yeast extract with/without $15 \mathrm{~g} \mathrm{~L}^{-1}$ agar), M63 minimum salts medium as previously described ${ }^{65}$ or 'spent' MHB medium. The carbon source in MHB and LB is an undefined mixture of peptides with minor traces of sugars. The spent MHB medium was prepared by removing wild-type cells from an overnight culture $\left(37^{\circ} \mathrm{C}, 200 \mathrm{rpm}\right.$ or $\left.1 \mathrm{~g}\right)$ by centrifugation and filtration through a $0.2 \mu \mathrm{m}$ filter. For spent MHB medium buffered at a certain $\mathrm{pH}$, the appropriate Good's buffering agent ${ }^{124}$ was added $(50 \mathrm{mM} 2-(N$-morpholino)ethanesulfonic acid for $\mathrm{pH} 5.5$ and 6 and $50 \mathrm{mM}$ piperazine- $N, N^{\prime}$-bis(2-ethanesulfonic acid) for $\mathrm{pH}$ 6.5) with/without potassium benzoate $(40 \mathrm{mM})$ and methylamine hydrochloride $(40 \mathrm{mM})$ to dissipate $\Delta \mathrm{pH}$. Next, the $\mathrm{pH}$ was adjusted using $\mathrm{HCl}$ or $\mathrm{KOH}$ and the medium was filter sterilized. For minimal media, carbon source stock solutions were prepared in demineralized water, adjusted to $\mathrm{pH} 7.0$ using $\mathrm{NaOH}$ or $\mathrm{HCl}$, respectively, and sterile filtered through a $0.2 \mu \mathrm{m}$ polyethersulfone (PES) filter. Glucose was added in a final concentration of $5 \mathrm{~g} \mathrm{~L}^{-1}$ and fumarate in a final concentration of $2 \mathrm{~g} \mathrm{~L}^{-1}$, respectively. Antibiotics, IPTG and arabinose were all prepared in demineralized water and sterile filtered through a $0.2 \mu \mathrm{m}$ PES filter. Bacterial cultures were cultivated either in $50 \mathrm{ml}$ medium in a $500 \mathrm{ml}$ or $10 \mathrm{ml}$ medium in a $100 \mathrm{ml}$ Erlenmeyer flask closed with a $38 \mathrm{~mm}$ silicone sponge closure (Bellco Glass) at $37^{\circ} \mathrm{C}, 300 \mathrm{rpm}$ and $5 \mathrm{~cm}$ throw $(5 \times g)$ or in $100 \mathrm{ml}$ medium in a $250 \mathrm{ml}$ Erlenmeyer flask closed using cellulose stoppers (VWR) at $37^{\circ} \mathrm{C}, 200 \mathrm{rpm}$ and $2.5 \mathrm{~cm}$ throw $(1 \times g)$. Overnight cultures were diluted 1:100 in fresh medium and further diluted $1: 100$ as soon as $\mathrm{OD}_{600}$ of 0.5 is reached to keep cells in the exponential growth phase.

Assessment of the antibiotic sensitivity. MICs, survival levels, and killing curves were determined according to the methods described previously $8,125,126$. For MIC determination an overnight culture was diluted in fresh MHB to an inoculum of $1 \times 10^{6}$ colony-forming units per milliliter $\left(\mathrm{CFU} \mathrm{ml} \mathrm{m}^{-1}\right)$ and incubated in a range of two-fold antibiotic dilutions for 16-20 h. After incubation, the lowest antibiotic concentration where no growth was observed using the absorbance at $595 \mathrm{~nm}$ (BioTek), we defined as the MIC. Alternatively, commercially available MIC test strips were used (Liofilchem).

Antibiotic survival level in the stationary phase is obtained by taking the ratio $\mathrm{CFU} \mathrm{ml} \mathrm{m}^{-1}$ after treatment (usually for $5 \mathrm{~h}$ ) and the total CFU ml ${ }^{-1}$ before antibiotic treatment (generally $1-5 \times 10^{9} \mathrm{CFU} \mathrm{ml}^{-1}$ ). CFU ml ${ }^{-1}$ were obtained by making serial dilutions, spiral plating, and semi-automatic quantification after 2 days of incubation at $37^{\circ} \mathrm{C}$ (EddyJet and Flash \& Grow). When using spent medium, stationary-phase cells were resuspended in an equal volume of the spent medium and treatment was started immediately.

The antibiotics that were used are all aminoglycosides or fluoroquinolones. We used these two classes as they are potent bactericidal drugs that furthermore can kill sensitive stationary phase cells and result in a biphasic killing pattern, indicative for the survival of persister cells. Furthermore, these antibiotics were used in our previous work which forms the basis of the current work ${ }^{8}$.

Assessment of antibiotic tolerance through cytometry upon nutrient shifts in M9 medium. Tolerance experiments upon nutrient shifts were always combined with ratiometric $\mathrm{pH}$ measurements. Cells were grown in $\mathrm{M} 9$ glucose medium containing ampicillin $\left(100 \mu \mathrm{g} \mathrm{ml}^{-1}\right)$ and arabinose $\left(20 \mathrm{~g} \mathrm{~L}^{-1}\right)$ with regular dilutions to maintain growth in the exponential phase. At $\mathrm{OD}_{600} 0.5, \mathrm{pH}$ was measured and persisters were induced by a switch to M9 fumarate medium. At multiple time points after the nutrient switch, samples were taken, and intracellular $\mathrm{pH}$ was determined as previously described. For determination of antibiotic tolerance, we treated the cells with amikacin $\left(4 \mathrm{~h}\right.$ at $\left.400 \mu \mathrm{g} \mathrm{ml}{ }^{-1}\right)$ at $0.5 \mathrm{~h}$ after the switch. As a control we blocked translation using a low dose of chloramphenicol $\left(25 \mu \mathrm{g} \mathrm{ml}^{-1}\right)$ added direct after the switch to fumarate. After $4 \mathrm{~h}$, amikacin treated cells were diluted 100-fold in LB and their ability to regrow was tracked using flow cytometry (for $3 \mathrm{~h}$, see refs. ${ }^{26,127}$ ). Tolerant cells resumed growth, became bigger and lost their pHluorin fluorescent intensity whereas non-growing cells retained their size and fluorescent intensity. The fraction of cells that did not resumed growth was determined by observing no cell size changes and no loss of fluorescence. Cells were measured every $30 \mathrm{~min}$ from 0 to $3 \mathrm{~h}$ after the transfer to LB medium. Fractions of non-recovering cells from the period of 2.5 and $3 \mathrm{~h}$ after transfer to LB were used to calculate the fractions of tolerant cells by subtracting the nondividing cells from the total cells added to the culture at the start.

Evolution experiments. Experimental evolution using the uropathogenic E. coli strain UTI89 was performed as before ${ }^{8,48}$. Briefly, parallel cultures were grown overnight to stationary phase in MHB $( \pm 18 \mathrm{~h})$, treated for $5 \mathrm{~h}$ with amikacin (400 $\mu \mathrm{g} \mathrm{ml}^{-1} ; 100-200$-fold MIC) to eliminate all non-persisters, washed three times in $\mathrm{MgSO}_{4}(10 \mathrm{mM})$ to remove antibiotics and diluted 1/100 into fresh MHB to allow another cycle of batch growth. A sigmoidal fit expected for the spread of a mutant in a haploid population was fitted to the data obtained under daily treatment:

$$
Y=z-\left(p_{0} \times\left(z-Z_{a}\right)\right) /\left(p_{0}+\left(1-p_{0}\right) \times\left(\frac{1}{1+s}\right)^{x}\right)
$$

with $s$ the selective advantage of the assumed mutant and $z$ and $Z_{a}$ are the logtransformed persister fraction of the ancestor and mutant. A horizontal line was fitted to the control data without treatment and compared to a straight line with an extra sum of squares $\mathrm{F}$ test to confirm the absence of change of phenotype. All fitting was done in GraphPad Prism 8 using the least squares method.

\section{Genome-wide next-generation sequencing. We detect mutations arising in} evolved populations or clones by genome-wide sequencing using Illumina's HiSeq platform. Genomic DNA was extracted by using the DNeasy Blood and Tissue Kit (Qiagen). Purity and concentration were assessed by Nanodrop, gel electrophoresis and Qubit. Average insert size of the prepared libraries was $\pm 300 \mathrm{bp}$ and sequencing was conducted at EMBL's Genecore in Germany, the VIB Nucleomics core in Belgium, Eurofins, or the Genomics core of UZ Gasthuisberg in Belgium. Raw data files have been deposited in the NCBI SRA database with following accession IDs: PRJNA498891 (populations of the lab strain), PRJNA270307 (clonal data of the lab strain), PRJNA498717 (uropathogenic populations), PRJNA498708 (uropathogenic clones) and PRJNA768774 (to check the $\Delta$ relASspoT deletion strains). The 100-150 bp paired-end output was analyzed using Qiagen's CLC Genomics Workbench version 11.0. Full details on the used workflow and parameters are available upon request. Briefly, reads were mapped to reference sequences after quality control, read trimming and filtering (NC_000913.3 for lab strain, NC_007946.1 and NC_007941.1 for the uropathogen UTI89). Mutation lists were obtained with CLC's low frequency variant detection tool. Further filtering of these lists was based on several quality features and prior experiences (scripts available upon request). Importantly, for clones, cutoff frequencies of $75 \%$ were used, while for population analysis, an initial cutoff frequency of 5\% was used while later on, this cutoff was dropped for the regions of the target genes specifically and data were filtered on minimum coverage only (coverage $>30$, forward \& reverse read count $>0$; script available upon request). Clonal results were furthermore confirmed using targeted Sanger sequencing with primers in Supplementary Data 4 . While we did not specifically remove intergenic or synonymous mutations, only 3 and 0 , respectively, were identified in the entire dataset at or above the cutoff frequency of $5 \%$. The perl-based software package Circos was used (http:// circos.ca/) to visualize data. Scripts and configuration files are available upon request. Mutations from the UTI background were added to figures, tables and data based on a pairwise alignment to the lab strain background.

For all the $\mathrm{Chi}^{2}$ tests to examine for significant enrichment, we used 200,000 replicates in Monte Carlo simulations to compute $p$-values and computed the null hypothesis as the condition in which mutations would be hitting randomly in the genome (Supplementary Fig. 1f), in genes (Supplementary Fig. 1g), in genes coding for membrane proteins (Supplementary Fig. 1h), in genes coding for innermembrane proteins (Supplementary Fig. 1i), in genes of the nuo operon (Fig. 2b), in nuo genes coding for the membrane-spanning subunit (Fig. 2c, Supplementary Fig. 3a, b). To do so, we used the genome-wide annotations in NC 000913: $4641652 \mathrm{bp}$ in total, $4089513 \mathrm{bp}$ of which are part of coding regions, $1040006 \mathrm{bp}$ of which code for membrane proteins, $822021 \mathrm{bp}$ of which code for inner-membrane proteins, $14673 \mathrm{bp}$ of which code for complex I of which $7110 \mathrm{bp}$ are for genes of the membrane subunit. For the membrane subunits, amino acids were scored to be part of either transmembrane helices or non-membrane-spanning loops based on predictions by Protter (wlab.ethz.ch/protter) ${ }^{128}$ and scored to be either hydrophobic or hydrophilic based on a scale computed before ${ }^{129}$ and a cutoff value of 0.6 .

Genomically repairing nuo-mutation. P1vir phage transduction was used to genomically revert the identified mutations in $n u o L, M$ and $N$ by using the $y f b P:: \mathrm{Km}^{\mathrm{R}}$ Keio mutant as donor strain as described elsewhere ${ }^{8,121}$.

Generating 3D structure of complex I from E. coli. No full protein structure exists for complex I of E. coli. To this end, we modeled the protein structure of the cytoplasmic domain (nuoBCDEFGI; uniprot IDs: P0AFC7, P33599, P0AFD1, P31979, P33602, P0AFD6) and membrane arm (nuoAHJKLMN; uniprot IDs: 
P0AFC3, P0AFD4, P0AFE0, P0AFE4, P33607, P0AFE8, P0AFF0) using the online SWISS modeling server (https://swissmodel.expasy.org/) ${ }^{130}$ with $6 \mathrm{~g} 2 \mathrm{j}$ and 4 he8 PDB entries, respectively, as templates (Global Model Quality Estimation of, respectively, 0.56 and 0.67 with $35.8 \%$ and $38.5 \%$ sequence identity). Next, an overall structure of complex I of $E$. coli was obtained by aligning the modeled structures of the cytoplasmic and membrane domains to the structure of complex I of Thermus thermophilus (PDB entry 4hea) ${ }^{51}$. To this end, chains G and L were used as anchors by aligning them through the structural alignment of multiple proteins (STAMP) tool ${ }^{131}$ in Visual Molecular Dynamics (VMD) ${ }^{132}$ with standard settings. Images were generated by the rendering capabilities of PyMOL ${ }^{133}$.

Purification of complex I and its variants. Complex I transcribed from the pBADnuo $n$ ио $F_{\text {His } 6}$ was expressed in, and purified from an E. coli strain lacking complex I and the alternative NADH dehydrogenase as performed before by the Friedrich group ${ }^{55}$. Site-specific mutagenesis through PCR with Q5 polymerase (NEB) on this construct using primers nuoL_FW and nuoL_RV, nuoM_FW and nuoM RV and nuoN_FW and nuoN_RV (Supplementary Data 4) generated the variants carrying the point mutations of interest. Specifically, after 1:100 dilution in baffled flasks containing autoinduction medium, BW25113 $\Delta n u o \Delta n d h$ with pBADnuo $n u F_{\mathrm{His}}$ was grown aerobically until late exponential phase $\left(\mathrm{OD}_{600}=3-4\right)$. From that point onwards, purification steps were carried out at $4^{\circ} \mathrm{C}$. Cells were harvested through centrifugation at $3000 \mathrm{~g}$ for $15 \mathrm{~min}$. Cell sediments were suspended with a Teflon-inglass homogenizer in a 5 -fold volume of buffer A ( $50 \mathrm{mM} \mathrm{NaCl}, 50 \mathrm{mM} \mathrm{MES} / \mathrm{NaOH}$, pH 6.0) with $0.1 \mathrm{mM}$ phenylmethylsulfonyl fluoride (PMSF) and few grains of Dnase I. Cells were disrupted by 4 passes through an Avestin Emulsiflex at $110 \mathrm{MPa}$ and cell debris was removed by low speed centrifugation at $9500 \mathrm{~g}$ for $20 \mathrm{~min}$. Next, membranes were separated from the supernatant by centrifugation at $257,000 \mathrm{~g}$ for $1 \mathrm{~h}$ and the membrane sediment was suspended in an equal volume of buffer $\mathrm{A}^{*}$ (buffer $\mathrm{A}+$ $5 \mathrm{mM} \mathrm{MgCl}_{2}$ ) with $0.1 \mathrm{mM}$ PMSF. Membrane suspensions were either directly used or stored as sediments at $-80^{\circ} \mathrm{C}$.

$n$-Dodecyl- $\beta$-D-maltopyranoside (DDM) was slowly added during $15 \mathrm{~min}$ to the membrane suspension until a final concentration of $2 \%$. After gentle stirring for $30 \mathrm{~min}$, insoluble material was removed at $250,000 \mathrm{~g}$ for $15 \mathrm{~min}$. Anion exchange chromatography on a Fractogel EMD TMAE Hicap (Merck) separated membrane proteins which were washed in buffer $A^{*}+0.1 \%$ DDM and eluted using buffer $B^{*}$ (Buffer $A^{*}$ with $350 \mathrm{mM} \mathrm{NaCl}$ and $0.1 \% \mathrm{DDM}$ ). Fractions with NADH/ ferricyanide oxidoreductase activity were pooled, corrected to contain $20 \mathrm{mM}$ imidazole and applied on Ni-IDA material (Invitrogen) for affinity chromatography. After washing with binding buffer ( $500 \mathrm{mM} \mathrm{NaCl}, 50 \mathrm{mM}$ MES/ $\mathrm{NaOH}, 0.1 \%$ DDM and $20 \mathrm{mM}$ imidazole ( $\mathrm{pH} 6.3$ ), fractions were eluted with $500 \mathrm{mM}$ imidazole. Fractions with NADH/ferricyanide oxidoreductase activity were pooled, washed three times with buffer $\mathrm{A}^{*}$ with $0.1 \% \mathrm{DDM}$, concentrated using ultrafiltration (Amicon Ultra-15, Millipore, $100 \mathrm{kDa}$ MWCO) and stored in aliquots at $-80^{\circ} \mathrm{C}$.

Protein concentration determination. Protein concentration was determined based on the biuret method using BSA as standard ${ }^{134}$. Concentration of purified complex I variants was measured by spectrometry (TIDAS II, J\&M Analytik) subtracting the absorbance at $310 \mathrm{~nm}$ from that at $280 \mathrm{~nm}$ and with an $\varepsilon$ of $763 \mathrm{mM}^{-1} \mathrm{~cm}^{-1}$.

NADH/ferricyanide oxidoreductase activity. NADH/ferricyanide oxidoreductase activity of membranes, fractions or purified complex I was determined by following the decrease in absorbance of ferricyanide at $410 \mathrm{~nm}$ in buffer A with $0.2 \mathrm{mM}$ NADH using an Ultrospec 1100 pro spectrophotometer (Pharmacia) ${ }^{56}$ and based on an $\varepsilon$ of $1 \mathrm{mM}^{-1} \mathrm{~cm}^{-1}$.

$\mathbf{O}_{\mathbf{2}}$ reduction assay. Reduction of $\mathrm{O}_{2}$ by electrons released from NADH by complex I was determined in buffer $\mathrm{A}^{*}$ on $5 \mu$ of membranes with a Clark-type oxygen electrode at $30^{\circ} \mathrm{C}$ (Hansatech). The reaction was started by adding $1.25 \mathrm{mM}$ NADH while $10 \mu \mathrm{M}$ piericidin A was used to inhibit the reaction.

Determination of purity and stability of complex I preparations. Initial production and stability of complex I were verified using polyacrylamide gel electrophoresis (PAGE). Under denaturing conditions, a sodium dodecyl sulfate (SDS) PAGE was run as described previously ${ }^{56}$ with a $3.9 \%$ stacking gel and a $10 \%$ separating gel. In addition, colorless native $(\mathrm{CN})$ PAGE was performed as described previously ${ }^{135}$ with a $3.5 \%$ stacking gel and a $4-13 \%$ gradient separating gel ( $\mathrm{pH}$ 6.0). For nitroblue tetrazolium (NBT) staining the gel was incubated for 5 min with $1 \mathrm{mg} \mathrm{ml}^{-1} \mathrm{NBT}$ in $100 \mathrm{mM}$ MOPS, pH 8 and the reaction was started by an addition of $100 \mu \mathrm{M}$ NADH.

In addition to PAGE, stability of purified complex I was verified with thermal shift assays. ThermoFAD measures the intrinsic fluorescence of flavin, a cofactor of complex I, to determine the unfolding temperature of the complex ${ }^{136}$. Briefly, $25 \mu \mathrm{L}$ of complex I (in buffer $\mathrm{A}, 1 \mu \mathrm{g} \mu \mathrm{L}^{-1}$ ) was heated in a CFX96 $\mathrm{qPCR}$ thermocycler (Bio-Rad) with fluorescence measurements at regular interval (ex. 470-500 nm, em. 523-543 nm). After subtracting blank data, a first derivative was calculated to obtain melting points as inflection points/maxima peaks. In addition, a Thermofluor-adapted assay ${ }^{137}$ made use of the increased fluorescent yield reaction of 7-diethylamino-3-(4'-maleimidylphenyl)-4-methylcoumarin (CPM) upon reaction with thiol groups that are released upon heating and unfolding. 300

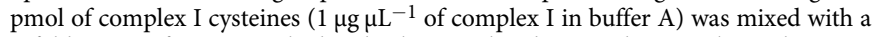
5 -fold excess of CPM, overlaid with silicone oil and assessed in a Perking Elmer LS55 fluorescence spectrometer (ex. $384 \mathrm{~nm}$, em. $470 \mathrm{~nm}$ ). A dose-response equation was fitted to the data in GraphPad Prism 8 using the least squares method:

$$
\left.Y=\text { Bottom }+(\text { Top }- \text { Bottom }) /\left(1+10^{\left(\left(\operatorname{LogEC}_{50}-X\right) * \text { Hillslope }\right)}\right)\right)
$$

A first derivative was calculated from this fit to obtain melting points as inflection points/maxima peaks ${ }^{57}$.

Reconstitution of complex I into liposomes. Purified complex I was reconstituted in liposomes at $4^{\circ} \mathrm{C}^{138}$. Briefly, complex I $\left(2 \mathrm{mg} \mathrm{ml}^{-1}\right)$ was mixed with a 4 -fold excess of E. coli polar lipids (w/w; extract from Avanti) which were dissolved in lipid-buffer $(5 \mathrm{mM} \mathrm{MES} / \mathrm{NaOH}, \mathrm{pH} 6.0)$ and DDM $\left(20 \mathrm{mg} \mathrm{ml}^{-1}\right)$ by sonication. The mixture was stirred gently for $10 \mathrm{~min}$ before BioBeads SM-2 (Bio-Rad) were added in an 8-fold excess, accounted for their binding capacity of and the presence of DDM in the sample and stirred gently for another $3 \mathrm{~h}$. The proteoliposomes were sedimented by centrifugation for $45 \mathrm{~min}$ at $150,000 \mathrm{~g}$, resuspended in proteoliposome buffer ( $5 \mathrm{mM}$ MES/ $\mathrm{NaOH}, \mathrm{pH} 6.0,50 \mathrm{mM} \mathrm{NaCl}, 5 \mathrm{mM} \mathrm{MgCl}_{2}$ ) by gentle pipetting, extruded with 31 passes through a $0.1 \mu \mathrm{m}$ polycarbonate membrane and used on the same day. The NADH/ferricyanide oxidoreductase activity before and after addition of 0.5\% DDM showed no significant differences between variants and wild type. The ratio of both activities was with on average 0.75 , indicating that $75 \%$ of the complex was oriented in the liposomes so that the NADH binding site was accessible from the buffer. Comparing to the NADH/ $\mathrm{FeCN}$ oxidoreductase activity of purified proteins, the average protein reconstitution efficiency was $21 \%$.

Preparation of ISOV vesicles. BW25113 $4 n u o \Delta n d h$ cells with pBADnuo nuo $F_{\text {His }}$ were induced and harvested as described above. Inside-out vesicles (ISOVs) containing the complex I variants and endogenous lipids and other proteins; were prepared at $4{ }^{\circ} \mathrm{C}$ from frozen cells following a slightly modified, previously described procedure ${ }^{139}$. Cell sediments were suspended in equal amount of washing buffer $\left(50 \mathrm{mM} \mathrm{KH}_{2} \mathrm{PO}_{4} / \mathrm{KOH}, 5 \mathrm{mM} \mathrm{MgSO}_{4}, \mathrm{pH}\right.$ 7.5), sedimented (10 min, 4,500 g), suspended 6:1 in lysis buffer (washing buffer with $1 \mathrm{mM}$ dithiothreitol, $0.1 \mathrm{mM}$ PMSF and some grains of Dnase I) and disrupted by a single pass through a $40 \mathrm{ml}$ French Pressure Cell at $55 \mathrm{MPa}$ (SLM-Aminco). Centrifugation removed debris $(20 \mathrm{~min}$ at $9500 \mathrm{~g}$ ). ISOVs were separated from the supernatant by two ultracentrifugation steps $(70 \mathrm{~min}$ and $15 \mathrm{~min}$ at $257,000 \mathrm{~g})$ and resuspended in washing buffer. ISOV vesicles were kept on $4^{\circ} \mathrm{C}$ and used on the same day.

Proton translocation and electron transfer activity. Proton translocation activity of complex I, either reconstituted in liposomes or in ISOVs, was measured by monitory quenching of fluorescence of 9-amino-6-chloro-2-methoxyacridine $(\text { ACMA })^{138}$. Briefly, proteoliposomes were incubated at $30^{\circ} \mathrm{C}$ for 1 min with $0.2 \mu \mathrm{M}$ ACMA and $60 \mu \mathrm{M}$ decyl-ubiquinone in ACMA buffer (5 mM MES/NaOH, $50 \mathrm{mM}$ $\mathrm{KCl}$ and $2 \mathrm{mM} \mathrm{MgCl}_{2} \mathrm{pH} 6.0$ ). The reaction was started by adding $100 \mu \mathrm{M} \mathrm{NADH}$ and ACMA fluorescence was followed on a Perking Elmer LS-55 fluorescence spectrometer through time (ex. $430 \mathrm{~nm}, \mathrm{em} .480 \mathrm{~nm}$ ). For measurements of proton transport using ISOVs, no decyl-ubiquinone was added. Similarly, without ACMA, $\mathrm{NADH} /$ decyl ubiquinone oxidoreduction was followed by monitoring the decreasing NADH concentration by spectrometry at $340 \mathrm{~nm}$ with an $\varepsilon$ of $6.3 \mathrm{mM}^{-1} \mathrm{~cm}^{-1}$ (TIDAS II, J\&M). As a control, the NADH/decyl ubiquinone oxidoreductase activity of empty liposomes was measured to be $15 \%$ of the activity of complex I-containing proteoliposomes.

Measurement of the electrical gradient in vivo. The electrical gradient across the membrane was determined using electrical-gradient dependent uptake of bis-(1,3dibarbituric acid)-trimethine oxonol $\left(\mathrm{DiBAC}_{4}(3)\right)^{8}$. Stationary phase cultures were incubated for $10-20 \mathrm{~min}$ with $10 \mu \mathrm{g} \mathrm{ml}^{-1} \mathrm{DiBAC}_{4}(3)$. Single-cell uptake was measured on a BD influx cytometer (ex. $488 \mathrm{~nm}$, em. 530/40 nm; 100,000 cells) and analyzed using FlowJo v10.3 (FlowJo, LLC). As control, samples were incubated for $1 \mathrm{~h}$ with $500 \mu \mathrm{M}$ of the protonophore carbonyl cyanide m-chlorophenyl hydrazone (CCCP) prior to staining with $\mathrm{DiBAC}_{4}(3)$.

Intracellular antibiotic accumulation measurement. To measure intracellular uptake of antibiotics, $50 \mathrm{ml}$ stationary phase cultures were incubated with amikacin $\left(100 \mu \mathrm{g} \mathrm{ml}^{-1}\right)$ or ofloxacin $\left(5 \mu \mathrm{g} \mathrm{ml}^{-1}\right)$ for $60 \mathrm{~min}$. Cells were separated from the extracellular solution by centrifugation through a 1:1.1 mixture of waterimpermeable silicone oils barrier (AR 20 and AR 200) and frozen at $-80^{\circ} \mathrm{C}$. For ofloxacin, cell sediments were suspended in $400 \mu \mathrm{l}$ of $0.1 \mathrm{M}$ glycine hydrochloride ( $\mathrm{pH} 3$ ) and lysed by incubating overnight at room temperature with light agitation Cellular debris was removed by centrifugation (10 min at $14,000 \mathrm{rpm}$ or $16,873 \mathrm{~g}$ ) and ofloxacin concentrations were analyzed by measuring fluorescence (ex. $292 \mathrm{~nm}, \mathrm{em} .496 \mathrm{~nm}$ ) using a Synergy Mx Microplate Reader (BioTek). Ofloxacin concentrations were determined from a calibration curve for concentrations $0-300 \mathrm{ng} \mathrm{ml}^{-1}$. For amikacin, an agar well diffusion bioassay was used ${ }^{140}$. Cell 
sediments were suspended in $750 \mu \mathrm{l}$ of PBS and lysed by incubation at $100^{\circ} \mathrm{C}$ for $7 \mathrm{~min}$. After centrifugation (10 min, 14,000 rpm or $16,873 g$ ) $100 \mu \mathrm{l}$ supernatants was pipetted in $8 \mathrm{~mm}$ diameter holes on MHB agar plates inoculated with Bacillus subtilis ATCC 6051 as the indicator organism. Plates were incubated for $24 \mathrm{~h}$ at $37^{\circ} \mathrm{C}$ after which the diameter of the inhibition zone was measured. Amikacin concentrations were deduced from a standard curve spanning a two-fold concentration range from 2 to $64 \mu \mathrm{g} \mathrm{ml}^{-1}$.

Ratiometric measurement of ATP:ADP ratios and cytoplasmic $\mathbf{p H}$. ATP:ADP ratio was determined using the ratiometric GFP-based biosensor Perceval ${ }^{64}$. The pRsetB-his7-Perceval plasmid was obtained from Addgene (plasmid \#20336) and the perceval gene was extracted by PCR using primer SPI-10577 and SPI-10578 (Supplementary Data 4). Next, Perceval was subcloned in $\mathrm{pBAD} / \mathrm{Myc}$-HisA, linearized through PstI and EcoRI digestion, using Gibson assembly (NEB) ${ }^{15}$. The 490/ $405 \mathrm{~nm}$ excitation fluorescence ratio $(\mathrm{em} .530 \mathrm{~nm})$ correlates with the ATP:ADP ratio and was determined using a Synergy MX Microplate reader (BioTek). Perceval's sensitivity towards intracellular $\mathrm{pH}$ was countered by resuspending cells that were growing on MHB supplemented with $2 \mathrm{~g} \mathrm{~L}^{-1}$ arabinose and $100 \mathrm{mg} \mathrm{L}^{-1}$ ampicillin, in M63 medium buffered at $\mathrm{pH} 7$ supplemented with potassium benzoate $(40 \mathrm{mM})$ and methylamine hydrochloride $(40 \mathrm{mM})$ before measurement.

Intracellular $\mathrm{pH}$ was measured with a GFP-based ratiometric sensor called pHluorin. We transformed the strains of interest with the pGFPR01 or pNTR-SDpHluorin vector in which pHluorin is expressed from the arabinose-inducible or IPTG-inducible promoters, respectively, $\mathrm{P}_{\mathrm{BAD}}$ and $\mathrm{P}_{\mathrm{tac}}{ }^{65,141}$. To induce pHluorin expression, cells were either grown on MHB medium supplemented with $2 \mathrm{~g} \mathrm{~L}^{-1}$ arabinose or $1 \mathrm{mM}$ IPTG or on M9 medium supplemented with $5 \mathrm{~g} \mathrm{~L}^{-1}$ glucose and $20 \mathrm{~g} \mathrm{~L}^{-1}$ arabinose with ampicillin $\left(100 \mathrm{mg} \mathrm{L}^{-1}\right)$ to select for the maintenance of the plasmid. Note that no ampicillin and arabinose was added after nutrient shift. Ratiometric fluorescent measurements to determine $\mathrm{pH}$ were performed by either a Synergy MX Microplate reader (BioTek; ex. 410 and $470 \mathrm{~nm} \pm 20 \mathrm{~nm}$; em. $530 \mathrm{~nm}$ $\pm 20 \mathrm{~nm}$ ) or a Spark plate reader (Tecan; ex. 380 and $470 \mathrm{~nm} \pm 20 \mathrm{~nm}$; em. $530 \mathrm{~nm}$ $\pm 20 \mathrm{~nm}$ ). Cells were either resuspended in M63 minimal salts medium or spun through HPLC spin filters (BaseClear) and resuspended in fresh M9 medium before measurement. A calibration curve was generated by measuring fluorescence ratios of cultures resuspended for at least $5 \mathrm{~min}$ in a range of buffers with different pHs supplemented with $40 \mathrm{mM}$ potassium benzoate and $40 \mathrm{mM}$ methylamine hydrochloride to dissipate the transmembrane $\mathrm{pH}$ gradient (see Martinez et al., 2012). The relation between the fluorescence ratio and the intracellular $\mathrm{pH}$ was described by fitting a Boltzmann sigmoid:

$$
\text { ratio }=\text { Bottom }+(\text { Top }- \text { Bottom }) /\left(1+\exp \left(\left(\mathrm{V} 50-\mathbf{p H}_{\mathrm{i}}\right) / \text { Slope }\right)\right) .
$$

Image analysis. Analysis of pictures of protein or DNA gels was done using Vilber's VisionCapt software for quantification and to estimate sizes.

Dynamic proteomics. For the dynamic proteomics analysis, we followed previously published protocols with minimum modification ${ }^{26,142}$. Briefly, cells were grown in M9 glucose medium with regular dilutions to maintain growth in exponential phase. At $\mathrm{OD}_{600} 0.45$, a proteomics sample was collected, and persisters were generated by a switch to M9 fumarate medium. At multiple time points after the nutrient switch, samples were collected, all containing $3 \times 10^{8}$ cells. They were centrifuged and washed with phosphate buffered saline $(2 \times)$ and then cell pellets were frozen in liquid nitrogen. Cell sediments were lysed in $2 \%$ sodium deoxycholate, $0.1 \mathrm{M}$ ammonium bicarbonate and disrupted by two sonication cycles (Hielscher ultrasonicator). BCA assay (Thermo Fisher Scientific) determined the protein concentration. Sample preparation involved: reduction with $5 \mathrm{mM}$ TCEP $\left(10 \mathrm{~min}, 95^{\circ} \mathrm{C}\right)$, alkylation with $10 \mathrm{mM}$ iodoacetamide $(30 \mathrm{~min}$, in the dark at room temperature), quenching with $12.5 \mathrm{mM} \mathrm{N}$-acetylcysteine, dilution with 0.1 $\mathrm{M}$ ammonium bicarbonate to a concentration of $1 \%$ sodium deoxycholate, digestion with trypsin (Promega; overnight with 50:1 protein:trypsin ratio), supplemented with $0.5 \%$ TFA and $50 \mathrm{mM} \mathrm{HCl}$, removal of precipitated sodium deoxycholate $\left(15 \mathrm{~min}\right.$ at $4{ }^{\circ} \mathrm{C}$ at $21,000 \mathrm{~g}$ ), desalting of peptides (C18 reversed phase spin columns; Macrospin, Harvard Apparatus), drying under vacuum, and storage at $-80^{\circ} \mathrm{C}$ until further processing. Samples were run on a dual pressure LTQOrbitrap Velos mass spectrometer connected to an electrospray ion source with peptide separation through an EASY nLC-1000 system (all Thermo Fisher Scientific) equipped with a RP-HPLC column $(75 \mu \mathrm{m} \times 45 \mathrm{~cm})$ packed with $\mathrm{C} 18$ resin (ReproSil-Pur C18-AQ; Dr. Maisch GmbH) using a linear gradient from $95 \%$ solvent A $(0.15 \%$ formic acid, $2 \%$ acetonitrile) to $28 \%$ solvent B ( $98 \%$ acetonitrile, $0.15 \%$ formic acid) over $90 \mathrm{~min}$ at $0.2 \mu \mathrm{min}^{-1}$. The acquisition mode obtained one high-resolution MS scan in the FT part at a resolution of 120,000 full width at half maximum (at $\mathrm{m} / \mathrm{z} 400$ ) followed by MS/MS scans in the linear ion trap of the 20 most intense ions. The charged state screening modus excluded unassigned and singly charged ions (dynamic exclusion duration: $20 \mathrm{~s}$; ion accumulation time: 300 $\mathrm{ms}$ (MS) and $50 \mathrm{~ms}$ (MS/MS)). The raw files were imported into the Progenesis LC-MS software (Nonlinear Dynamics, Version 4.0) and analyzed using the default settings. MS/MS-data were exported in mgf format and searched against a decoy database of the forward and reverse sequences of the predicted proteome from $E$. coli (Uniprot, download date: 15/6/2012, total of 10,388 entries) using MASCOT. In the search criteria, full tryptic specificity was required (after lysine or arginine residues), three missed cleavages were allowed, carbamidomethylation (C) was set as fixed modification, oxidation (M) as variable modification and mass tolerance was $10 \mathrm{ppm}$ for precursor ions and 0.6 Da for fragment ions. Results were imported into Progenesis, and the false discovery rate (FDR) was set to $1 \%$. The final protein lists containing the cumulative peak areas of all identified peptides for each protein, respectively, were exported from Progenesis LC-MS and further statically analyzed using an in-house developed R script (SafeQuant) ${ }^{142}$. The raw mass spectrometry data is available through the ProteomeXchange Consortium (ID: PXD029006).

For the analysis, we normalized our data relative to a 2 -fold difference in protein concentrations between all the analyzed conditions with the WT at start (before nutrient shift) as a reference. Next, we applied unscaled, two-dimensional principal component analysis using the FactoMineR package (R). For this, we used a previously published and analyzed dataset (from Schmidt et al. ${ }^{69}$ and Fig. $4 \mathrm{a}$ in Radzikowski et al. ${ }^{26}$, ProteomeXchange IDs: PXD000498 and PXD001968, respectively) to generate the two dimensions that constitute the PCA space. We plotted our data on this space to compare with previously found trends.

Radioactive assessment of translation activity. To probe protein synthesis, we opted to follow the incorporation of radioactive $\mathrm{L}-\left(4,5-{ }^{3} \mathrm{H}\right)$-leucine during stationary phase in complex MHB medium $( \pm 18 \mathrm{~h}$ after 1:100 dilution as done during the evolution experiment) instead of following induction of a fluorescent protein as done before ${ }^{8}$ as the latter might be prone to biased results. Specifically, L- $\left(4,5-{ }^{3} \mathrm{H}\right)$ leucine was added to stationary phase cultures at $2.5 \mu \mathrm{Ci} \mathrm{ml}^{-1}$ and further incubated shaking at $37^{\circ} \mathrm{C}$. As control, chloramphenicol was additionally added at $64 \mu \mathrm{g} \mathrm{ml}^{-1}$, which fully abrogated protein synthesis (data not shown). Over the course of $3 \mathrm{~h}$, samples were taken on an hourly basis and precipated in ice-cold trichloric acid (TCA; 10\%). Precipitates were washed twice with ice-cold destilled water and added to scintillation liquid (Ultima-Flo M, Perking Elmer). Radioactive signal was measured as counts per minute with a Hidex 300SL scintillation counter.

HPLC-UV detection and quantification of ppGpp. Solutions containing ppGpp (Jena Biosciences) and products of its hydrolysis were analyzed using a HPLC-UV method measuring absorbance at $260 \mathrm{~nm}$. A PL-SAX anion exchange column (PLSAX $1000 \AA 8 \mu \mathrm{m}, 50 \times 4.6 \mathrm{~mm}$, Agilent) was used for sample separation at $60^{\circ} \mathrm{C}$. Two methods were used: either (1) 0-2 min linear gradient from $80 \%$ to $70 \% \mathrm{~A}$, 2-4 min linear gradient from $70 \%$ to $0 \% \mathrm{~A}, 4-9.5 \mathrm{~min} 0 \% \mathrm{~A}$, at $9.5 \mathrm{~min}$ step change to $80 \%$ kept until $10 \mathrm{~min}$; or (2) a $2.5 \mathrm{~min}$ (or $5 \mathrm{~min}$, respectively) isocratic flow of B; A: $0.01 \mathrm{M} \mathrm{K}_{2} \mathrm{HPO}_{4}$, pH 2.6; B: $0.5 \mathrm{M} \mathrm{K}_{2} \mathrm{HPO}_{4}, \mathrm{pH}$ 3.5. The flow rate was $1 \mathrm{ml} \mathrm{min}{ }^{-1}$ in (1) or $1.2 \mathrm{ml} \mathrm{min}^{-1}$ in (2).

Spontaneous ppGpp hydrolysis assays. Spontaneous (i.e. non-enzymatic) ppGpp hydrolysis can occur at low pH once the two distal phosphate groups of ppGpp are deprotonated and a positively charged divalent metal ion coordinates between the phosphate groups (Supplementary Fig. 6a). Spontaneous hydrolysis assays were performed in a buffer containing $0.05 \mathrm{M}$ Tris, $0.09 \mathrm{M}$ sodium formate and $0.025 \mathrm{M}$ ammonium acetate, with adjusted to $\mathrm{pH}$ values and manganese concentrations as indicated. ppGpp (Jena Biosciences) was added in a final concentration of $1 \mathrm{mM}$ sampled by HPLC at $37^{\circ} \mathrm{C}$. In order to prevent evaporation, the mixture was covered with PCR mineral oil. $2 \mu \mathrm{L}$ of the reaction volume was injected at different points in time and the amount of ppGpp, as well as its hydrolysis products, were quantified by comparison to pure standards at different concentrations. Consistent with computational estimations of the $\mathrm{pK}_{\mathrm{a}}$ values of the two distal phosphate groups of $\mathrm{ppGpp}^{143}$ (i.e. 6.5 and 6.8), we found that spontaneous ppGpp hydrolysis is high at $\mathrm{pH}$ of 8 , and absent at $\mathrm{pH}$ of 7 , with a sharp change in velocity around $\mathrm{pH} 7.5$, and only occurs in the presence of divalent metal cations like manganese (Supplementary Fig. $6 \mathrm{~b}-\mathrm{d}, \mathrm{g}$ ). Thus, at $\mathrm{pH}$ values found in persisters, spontaneous ppGpp hydrolysis is inhibited (Fig. 5a, b).

Overproduction and purification of 6His-SpoT. To identify whether an enzymebased catalysis of ppGpp hydrolysis (potentially also $\mathrm{pH}$ inhibited) is partially responsible for the previously observed increase in ppGpp levels in persisters ${ }^{26}$, we focused on the enzymes synthesizing (RelA, SpoT) and hydrolyzing ppGpp (SpoT). As a deletion of relA had no effect on persister fractions, we had previously inferred that SpoT must be the player responsible for increased ppGpp levels in nutrient shift-induced persisters ${ }^{26}$. To further identify whether the ppGpp increase is due to an increased SpoT synthetase activity or an decreased SpoT hydrolysis activity, we initially analyzed a strain carrying a SpoT variant, which is defective in (p)ppGpp synthesis, but not in hydrolysis activity (SpoT-E319Q) ${ }^{144}$. After a glucose-tofumarate nutrient shift, we found the same fraction of persisters as in the wild type (Supplementary Fig. 6e), suggesting that an inhibition of the hydrolase activity of SpoT must be responsible for the increased ppGpp levels in persisters formed upon a nutrient shift. To test whether, next to the spontaneous ppGpp hydrolysis, also the SpoT hydrolase activity is pH dependent, we aimed to purify SpoT, whose production and purification is notoriously difficult ${ }^{145,146}$. While the protein mostly resided in the insoluble protein fraction, we could purify minute amounts of soluble protein after a short induction time using either a Tris-based buffer (Supplementary Fig. 6h) or a cytosolic buffer (phosphate-based buffer, see below and Supplementary Fig. 6j). 
SpoT was expressed with an N-terminal His-tag using plasmid pETM11-SpoT. pETM11-SpoT was generated by amplification of spoT using primer pair spoT_NcoI_s and spoT_NotI_stop_as (Supplementary Data 4) using genomic DNA from E. coli BW25113 as template. Subsequently the DNA fragment was cloned into pETM11 (EMBL Heidelberg) using NcoI and NotI restriction sites. Successful insertion was confirmed by restriction and sequencing analyses. The plasmid was transformed into E. coli BL21 (DE3) pLysS and selected on LB plates containing kanamycin and chloramphenicol.

E. coli strain BL21 (DE3) pLyS transformed with plasmid pETM11-SpoT was grown aerobically at $37^{\circ} \mathrm{C}$ in $100 \mathrm{ml} \mathrm{LB}$ medium in a $1000 \mathrm{ml}$ flask supplemented with kanamycin and chloramphenicol (both at $50 \mu \mathrm{g} \mathrm{ml}^{-1}$ ). Gene expression was induced at $\mathrm{OD}_{600}=0.5$ by adding $0.5 \mathrm{mM}$ isopropylthio- $\beta$-galactoside (IPTG) and cells were grown for $1 \mathrm{~h}$ at $37^{\circ} \mathrm{C}$. Cells were harvested, washed with buffer $(50 \mathrm{mM}$ Tris/HCl pH 7.5, 10\% glycerol) and disrupted by passage through a Cell disruptor (Constant Cell Disruption Systems, Northants, UK) at 25 bar and $4{ }^{\circ} \mathrm{C}$ in disruption buffer ( $50 \mathrm{mM}$ Tris/ $\mathrm{HCl} \mathrm{pH} \mathrm{7.7,} \mathrm{10 \%} \mathrm{(v/v)} \mathrm{glycerol,} 300 \mathrm{mM} \mathrm{NaCl}, 50$ $\mathrm{mM} \mathrm{KCl}, 1 \mathrm{mM}$ dithiothreitol, $0.5 \mathrm{mM}$ PMSF, and $0.03 \mathrm{mg} / \mathrm{ml}$ (w/v) DNase). After removal of intact cells and cell debris by centrifugation $\left(3000 \mathrm{~g}, 10 \mathrm{~min}, 4^{\circ} \mathrm{C}\right)$, the cytosol was incubated over night at $4{ }^{\circ} \mathrm{C}$ with pre-equilibrated Ni-Sepharose (with $50 \mathrm{mM}$ Tris/HCl pH 7.7, 10\% (v/v) glycerol, $300 \mathrm{mM} \mathrm{NaCl}, 10 \mathrm{mM} \mathrm{MgCl} 2,2 \mathrm{mM}$ $\beta$-mercaptoethanol, $10 \mathrm{mM}$ imidazole). Unbound protein was removed by washing with equilibration buffer and 6His-SpoT was eluted from the column by increasing imidazole concentrations up to $250 \mathrm{mM}$. Obtained purified protein degraded already at $4{ }^{\circ} \mathrm{C}$ (Supplementary Fig. 6i).

To obtain more stable protein, purification was attempted with cytosolic buffer. Cells were cultivated as described above and disrupted in cytosolic buffer $\mathrm{pH} 7.5$ $\left(6 \mathrm{mM} \mathrm{KH}_{2} \mathrm{PO}_{4}, 14 \mathrm{mM} \mathrm{KH}_{2} \mathrm{PO}_{4}, 140 \mathrm{mM} \mathrm{KCl}, 5.5 \%\right.$ glucose, $\left.10 \mathrm{mM} \mathrm{NaCl}\right)$ containing $0.03 \mathrm{mg} / \mathrm{ml}$ DNAse $(\mathrm{w} / \mathrm{v})$ and $0.5 \mathrm{mM}$ PMSF. Removal of intact cells and purification of $6 \mathrm{His}-\mathrm{SpoT}$ was performed as described above, but with use of cytosolic buffers containing imidazole. Note that $\mathrm{pH}$ of the buffers was re-adjusted after imidazole addition using phosphoric acid and $\mathrm{KOH}$, respectively. For purification of SpoT at different $\mathrm{pH}$, cells were disrupted in cytosolic buffer $\mathrm{pH}$ 7.5, bound to Ni-Sepharose and washed with buffer $\mathrm{pH}$ 7.5, and eluted with buffers adjusted to the corresponding $\mathrm{pH}$ and containing increasing imidazole concentrations. Protein content in samples was analyzed by SDS PAGE (12\%) and Bradford protein assay ${ }^{147}$. The purified protein was slightly more stable than with the Tris-based buffer, but ppGpp hydrolysis activity declined strongly over time (Supplementary Fig. 6c, d). In addition, the amount of protein was rather low $\left(0.3 \mathrm{mg} \mathrm{ml}^{-1}\right)$ and attempts to concentrate the samples or exchange buffers remained unsuccessful, rendering systematic assessment of the $\mathrm{pH}$ dependency of SpoT hydrolysis impossible in this way.

Co-expression and purification of encapsulin and SpoT. To obtain purified and stable protein, we followed an alternative approach, where we expressed SpoT in nanocages, formed as icosahedral capsids of 60 monomers of a small bacterial protein (encapsulin), which can protect the cargo protein from aggregation and degradation ${ }^{148}$. Nanocages or encapsulins are proteins that form macromolecular assemblies, resembling viral capsids 148 . Such capsids usually contain DyPperoxidase or ferritin-like proteins ${ }^{148,149}$. Efficient encapsulation in vivo is possible due to the presence of $\sim 30$ AA C-terminal signal peptide, which interacts with inner surface of encapsulin ${ }^{148}$. Genes encoding for encapsulin and cargo enzymes are found together in an operon, since encapsulin assembly and cargo loading is a concerted process ${ }^{148}$. Here, we used a newly discovered robust encapsulin from Mycolicibacterium hassiacum, for which it was demonstrated to increase the stability of foreign cargo enzymes ${ }^{150}$ and which enabled the characterization of the activity of the encapsulated and thus stabilized enzymes, as the nanocages contain three types of pores (5-9 $\AA$ ), which allow small substrates to enter or leave the capsule $^{148,149,151}$.

For co-expression of SpoT and encapsulin, spoT was amplified using forward and reverse primer (Supplementary Data 4). BsaI sites are underlined and specific overhangs are indicated in bold. spoT was cloned in a pENC vector based on PET28 with a changed origin of replication to $\mathrm{p} 15 \mathrm{~A}$ in order to be compatible with $\mathrm{pBAD}$ vector, and with an introduced BsaI restriction sites. These changes have been introduced using Gibson assembly. Briefly, the p15A ori was amplified from pEVOL-pAzF (Addgene plasmid \#31186) and the pET-28a(+) vector was amplified excluding pBR322 ori. These two fragments were assembled using NEB's Gibson assembly mix, with all the primers designed using Geneious software (Gibson cloning option; vector map available on request). The gene encoding SpoT was cloned into pENC in such way that, when expressed, it contains a N-terminal 6xHis-tag and also C-terminal fusion tag of 30 amino acids from DyP-peroxidase from the same operon as the original encapsulin gene ${ }^{150}$. EncMh encapsulin was provided by expression from $\mathrm{pBAD}$-EncMh, obtained by cloning the codonoptimized gene encoding EncMh (GenScript) between the NdeI and HindIII restriction site of a $\mathrm{pBAD}-\mathrm{NK}$ vector ${ }^{150}$. After sequencing, both the vectors were co-transformed into E. coli BL21-AI strain (ThermoFisher) and selected on LB plates containing ampicillin, kanamycin and tetracyclin.

E. coli strain BL21-AI transformed with plasmids $\mathrm{pBAD}-\mathrm{EncMh}$ and pENCspoT was grown aerobically at $37^{\circ} \mathrm{C}$ in $200 \mathrm{ml} \mathrm{LB}$ containing ampicillin and kanamycin (both $50 \mu \mathrm{g} \mathrm{ml}^{-1}$ ) in a $1000 \mathrm{ml}$ baffled flask. At $\mathrm{OD}_{600}=0.5$ expression of spoT was induced by $1 \mathrm{mM}$ IPTG (final concentration) and expression of encapsulin by addition of $0.2 \%$ arabinose (final concentration) and cells were incubated for another $16-18 \mathrm{~h}$ at $30^{\circ} \mathrm{C}$. Cells were collected by centrifugation for $20 \mathrm{~min}$ at $6000 \mathrm{rpm}(3099 \mathrm{~g})$ and $4^{\circ} \mathrm{C}$. Sonication was carried out in $50 \mathrm{mM}$ TrisHCl pH 7.5 with $150 \mathrm{mM} \mathrm{NaCl}$. As it can happen that cargo protein is expressed in higher amount than what can be encapsulated it is necessary to remove excess. Clarified cell-free extract was loaded on pre-equilibrated $\mathrm{Ni}$ Sepharose column to remove excess of cargo protein which contains 6xHis-tag. Since encapsulin does not contain any $6 \mathrm{xHis}$ tag and due to its size, it comes in flow-through fraction, together with any cargo protein it may contain. Flowthrough fraction was mixed on ice 1:0.75 with 10\% PEG8000 in $100 \mathrm{mM} \mathrm{Tris} / \mathrm{HCl}$ $\mathrm{pH} 7.5$ with $2 \mathrm{M} \mathrm{NaCl}$ and left on a nutating shaker at $4^{\circ} \mathrm{C}$ for $8 \mathrm{~h}$. Precipitated proteins were collected by centrifugation for $30 \mathrm{~min}$ at $4000 \mathrm{rpm}(1377 \mathrm{~g})$ and $4{ }^{\circ} \mathrm{C}$ Sediment was resuspended in $20 \mathrm{ml}$ of $50 \mathrm{mM}$ TrisHCl pH 7.5 and left overnight on a nutating shaker at $4{ }^{\circ} \mathrm{C}$ to re-dissolve completely. Re-dissolved ENC-spoT was aliquoted, flash-frozen in liquid nitrogen and stored at $-80^{\circ} \mathrm{C}$. Following this approach, we could obtain co-purified encapsulin with stable SpoT at $3 \mathrm{mg} \mathrm{m}^{-1}$ (Supplementary Fig. 6m).

Enzymatic ppGpp hydrolysis assay. We used both the encapsulated SpoT and regularly purified SpoT to characterize the $\mathrm{pH}$ dependency of its ppGpp hydrolysis activity. While the product of the spontaneous hydrolysis reaction leads to GTP, experiments with the limited amounts of the regularly purified SpoT indicated that SpoT hydrolyzes ppGpp to GDP, and that manganese ions are necessary for hydrolysis (Supplementary Fig. 6g), consistent with earlier reported requirements of Rel/Spo homologues for hydrolysis ${ }^{152,153}$. Enzymatic ppGpp hydrolysis assays with 6 His-SpoT and encapsulin SpoT were performed in cytosolic buffer containing $250 \mathrm{mM}$ imidazole (and $0.1 \%$ triton-X100 in case of encapsulin SpoT) adjusted to the corresponding $\mathrm{pH}$ with a final protein concentration of 0.1 and $0.5 \mathrm{mg} \mathrm{ml}^{-1}$, respectively. Unless stated otherwise, manganese was added $(1 \mathrm{mM})$ and the reaction was started by adding ppGpp $(1 \mathrm{mM})$. Samples were incubated at $25^{\circ} \mathrm{C}$ and, at different time points, aliquots were taken in which the reaction was stopped by adding EDTA $(10 \mathrm{mM})$. Two microliters of each aliquot was used for HPLC analysis as described above. Here, similar to the results of the spontaneous hydrolysis, we found that the hydrolysis activity is strongly $\mathrm{pH}$-dependent: hydrolysis starts at $\mathrm{pH}$ values higher than 7.0 , sharply increases around $\mathrm{pH} 7.5$ and reaches a maximum at around $\mathrm{pH} 8.3$, above which the hydrolysis activity drops again likely due to enzyme denaturation (Supplementary Fig. 6f). A control experiment with an empty capsule sample at $\mathrm{pH} 8$ revealed no ppGpp hydrolysis and few limited experiments with the minute amounts of regularly purified SpoT confirmed the $\mathrm{pH}$ dependency of hydrolysis (Supplementary Fig. 6f). Thus, also the ppGpp hydrolysis of SpoT is strongly $\mathrm{pH}$-dependent, with hardly any activity around $\mathrm{pH} 7.0$ and very much increased rates above $\mathrm{pH}$ 7.5. As persister cells have a cytoplasmic $\mathrm{pH}$ below 7.5 (Fig. 5a, b), we conjecture that the cytoplasmic $\mathrm{pH}$, via inhibition of ppGpp hydrolysis, is a major regulator of ppGpp levels upon strong perturbation of metabolic homeostasis.

Statistics. Data were analyzed using either GraphPad Prism 8 or R in the RStudio environment. Relevant information regarding the statistical tests was added to each figure caption where appropriate. In Fig. 1b, c, Supplementary Figs. If-I and 2a, b, $\mathrm{Chi}^{2}$ goodness-of-fit tests with 200,000 Monte Carlo simulations to compute p-values were used to compare the observed distribution of our mutations counts in various categories to the expected probabilities based on random occurring mutations. More detailed information can be found under the section explaining the genome-wide sequencing analyses and $\mathrm{Chi}^{2}$ statistics are reported in Supplementary Data 4. For ANOVAs and linear (mixed) models, assumptions of homoscedasticity and normality of the residuals were checked visually comparing fitted value with residuals and comparing predicted with actual residuals. Where relevant, the Brown-Forsythe test was used to test whether SDs were equal, the Variance Inflation Factor was used to estimate the degree of multicollinearity and a $\mathrm{Chi}^{2}$ test was performed to test the effectiveness of including random effects/ matching. Post hoc tests (posttest) were performed to make and correct for multiple comparisons. All tests for which it applies were two-tailed. Throughout the manuscript, a significance level cutoff of alpha $=0.05$ was used. For visual purposes, asterisks denote significance levels as defined in the figure captions and each exact $p$ value can be found in Supplementary Data 4. In addition, test statistics and degrees of freedom are reported in Supplementary Data 4.

Reporting summary. Further information on research design is available in the Nature Research Reporting Summary linked to this article.

\section{Data availability}

The NGS Sequencing data generated in this study have been deposited in the SRA database under accession codes PRJNA498891, PRJNA498708, PRJNA498717, PRJNA270307, and PRJNA768774. Mass spectrometry raw data files have been deposited to the ProteomeXchange Consortium via the PRIDE partner repository under dataset identifier PXD029006. The data underlying all the figures generated in this study are provided in the Source Data file. Mass spectrometry data used in this study are available via the ProteomeXchange Consortium via the PRIDE partner repository under the dataset identifiers PXD000498 and PXD001968. Sequence data used in this study are 
available in the NCBI nucleotide database (NC_000913.3, NC_007946.1, NC_007941.1), Uniprot database (P0AFC3, P0AFC7, P33599, P0AFD1, P31979, P33602, P0AFD4, P0AFD6, P0AFE0, P0AFE4, P33607, P0AFE8, P0AFF0), PDB database (6g2j, 4he8) and PubChem database (135398637). Source data are provided with this paper.

Received: 23 March 2020; Accepted: 4 January 2022;

Published online: 27 January 2022

\section{References}

1. Balaban, N. Q. et al. Definitions and guidelines for research on antibiotic persistence. Nat. Rev. Microbiol. 17, 441-448 (2019).

2. Cohen, N. R., Lobritz, M. A. \& Collins, J. J. Microbial persistence and the road to drug resistance. Cell Host Microbe 13, 632-642 (2013).

3. Van den Bergh, B., Fauvart, M. \& Michiels, J. Formation, physiology, ecology, evolution and clinical importance of bacterial persisters. FEMS Microbiol. Rev. 41, 1-33 (2017).

4. Dewachter, L., Fauvart, M. \& Michiels, J. Bacterial heterogeneity and antibiotic survival: Understanding and combatting persistence and heteroresistance. Mol. Cell 76, 255-267 (2019).

5. Fisher, R. A., Gollan, B. \& Helaine, S. Persistent bacterial infections and persister cells. Nat. Rev. Microbiol. 15, 453-464 (2017).

6. Fauvart, M., De Groote, V. N. \& Michiels, J. Role of persister cells in chronic infections: clinical relevance and perspectives on anti-persister therapies. $J$. Med. Microbiol. 60, 699-709 (2011).

7. Fridman, O., Goldberg, A., Ronin, I., Shoresh, N. \& Balaban, N. Q. Optimization of lag time underlies antibiotic tolerance in evolved bacterial populations. Nature 99, 1-9 (2014).

8. Van den Bergh, B. et al. Frequency of antibiotic application drives rapid evolutionary adaptation of Escherichia coli persistence. Nat. Microbiol. 1, 16020 (2016).

9. Mechler, L. et al. A novel point mutation promotes growth phase-dependent daptomycin tolerance in Staphylococcus aureus. Antimicrob. Agents Chemother. 59, 5366-5376 (2015).

10. Michiels, J. E., Van den Bergh, B., Verstraeten, N. A., Fauvart, M. \& Michiels, $\mathrm{J}$. In vitro emergence of high persistence upon periodic aminoglycoside challenge in the ESKAPE pathogens. Antimicrob. Agents Chemother. 60, 4630-4637 (2016).

11. Levin-Reisman, I. et al. Antibiotic tolerance facilitates the evolution of resistance. Science 355, 826-830 (2017).

12. Windels, E. M. et al. Bacterial persistence promotes the evolution of antibiotic resistance by increasing survival and mutation rates. ISME J. 13, 1239-1251 (2019).

13. Sebastian, J. et al. De novo emergence of genetically resistant mutants of Mycobacterium tuberculosis from the persistence phase cells formed against antituberculosis drugs in vitro. Antimicrob. Agents Chemother. 61, e01343-16 (2016).

14. Rotem, E. et al. Regulation of phenotypic variability by a threshold-based mechanism underlies bacterial persistence suppl info. Proc Natl Acad Sci USA 107, 1-5 (2010).

15. Wilmaerts, D. et al. The persistence-inducing toxin HokB forms dynamic pores that cause ATP leakage. mBio 9, 1-12 (2018).

16. Cheverton, A. M. et al. A Salmonella toxin promotes persister formation rhrough acetylation of tRNA. Mol. Cell 63, 86-96 (2016).

17. Dörr, T., Vulić, M. \& Lewis, K. Ciprofloxacin causes persister formation by inducing the TisB toxin in Escherichia coli. PLoS Biol. 8, e1000317 (2010).

18. Verstraeten, N. A. et al. Obg and membrane depolarization are part of a microbial bet-hedging strategy that leads to antibiotic tolerance. Mol. Cell $\mathbf{5 9}$, 9-21 (2015)

19. Schumacher, M. A. et al. HipBA-promoter structures reveal the basis of heritable multidrug tolerance. Nature 524, 59-64 (2015).

20. Jurenas, D. et al. AtaT blocks translation initiation by $\mathrm{N}$-acetylation of the

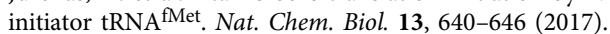

21. Wilmaerts, D. et al. HokB monomerization and membrane repolarization control persister awakening. Mol. Cell 75, 1031-1042.e4 (2019).

22. Fung, D. K. C., Chan, E. W. C., Chin, M. L. \& Chan, R. C. Y. Delineation of a bacterial starvation stress response network which can mediate antibiotic tolerance development. Antimicrob. Agents Chemother. 54, 1082-1093 (2010).

23. Nguyen, D. et al. Active starvation responses mediate antibiotic tolerance in biofilms and nutrient-limited bacteria. Science 334, 982-986 (2011).

24. Korch, S. B., Henderson, T. A. \& Hill, T. M. Characterization of the hipA7 allele of Escherichia coli and evidence that high persistence is governed by (p) ppGpp synthesis. Mol. Microbiol. 50, 1199-1213 (2003).
25. Gaca, A. O., Colomer-Winter, C. \& Lemos, J. A. Many means to a common end: The intricacies of (p)ppGpp metabolism and its control of bacterial homeostasis. J. Bacteriol. 197, 1146-1156 (2015).

26. Radzikowski, J. L. et al. Bacterial persistence is an active $\sigma^{\mathrm{S}}$ stress response to metabolic flux limitation. Mol. Syst. Biol. 12, 882 (2016).

27. Amato, S. M. \& Brynildsen, M. P. Persister heterogeneity arising from a single metabolic stress. Curr. Biol. 25, 2090-2098 (2015).

28. Amato, S. M., Orman, M. A. \& Brynildsen, M. P. Metabolic control of persister formation in Escherichia coli. Mol. Cell 50, 475-487 (2013).

29. Germain, E., Castro-Roa, D., Zenkin, N. \& Gerdes, K. Molecular mechanism of bacterial persistence by HipA. Mol. Cell 52, 248-254 (2013).

30. Kaspy, I. et al. HipA-mediated antibiotic persistence via phosphorylation of the glutamyl-tRNA-synthetase. Nat. Commun. 4, 1-7 (2013).

31. Stokes, J. M., Lopatkin, A. J., Lobritz, M. A. \& Collins, J. J. Bacterial metabolism and antibiotic efficacy. Cell Metab. 30, 251-259 (2019).

32. Radzikowski, J. L., Schramke, H. \& Heinemann, M. Bacterial persistence from a system-level perspective. Curr. Opin. Biotechnol. 46, 98-105 (2017).

33. Spoering, A. L., Vulić, M. \& Lewis, K. GlpD and PlsB participate in persister cell formation in Escherichia coli. J. Bacteriol. 188, 5136-5144 (2006).

34. Girgis, H. S. H., Harris, K. \& Tavazoie, S. Large mutational target size for rapid emergence of bacterial persistence. Proc. Natl Acad. Sci. USA 109, 12740-12745 (2012)

35. Hansen, S., Lewis, K. \& Vulić, M. Role of global regulators and nucleotide metabolism in antibiotic tolerance in Escherichia coli. Antimicrob. Agents Chemother. 52, 2718-2726 (2008).

36. Wang, W. et al. Transposon mutagenesis identifies novel genes associated with Staphylococcus aureus persister formation. Front. Microbiol. 6, 1-9 (2015).

37. $\mathrm{Ma}, \mathrm{C}$. et al. Energy production genes $s u c B$ and $u b i F$ are involved in persister survival and tolerance to multiple antibiotics and stresses in Escherichia coli. FEMS Microbiol. Lett. 303, 33-40 (2010).

38. Kotte, O., Volkmer, B., Radzikowski, J. L. \& Heinemann, M. Phenotypic bistability in Escherichia coli's central carbon metabolism. Mol. Syst. Biol. 10, 736-736 (2014).

39. Balaban, N. Q., Merrin, J., Chait, R., Kowalik, L. \& Leibler, S. Bacterial persistence as a phenotypic switch. Science 305, 1622-1625 (2004).

40. Gutierrez, A. et al. Understanding and sensitizing density-dependent persistence to quinolone antibiotics. Mol. Cell 68, 1147-1154.e3 (2017).

41. $\mathrm{Pu}$, Y. et al. ATP-dependent dynamic protein aggregation regulates bacterial dormancy depth critical for antibiotic tolerance. Mol. Cell 73, 143-156.e4 (2019).

42. Conlon, B. P. et al. Persister formation in Staphylococcus aureus is associated with ATP depletion. Nat. Microbiol. 1, 16051 (2016).

43. Shan, Y. et al. ATP-dependent persister formation in Escherichia coli. mBio 8 , e02267-16 (2017)

44. Orman, M. A. \& Brynildsen, M. P. Dormancy is not necessary or sufficient for bacterial persistence. Antimicrob. Agents Chemother. 57, 3230-3239 (2013).

45. Ray, J. C. J. et al. Cellular growth arrest and persistence from enzyme saturation. PLoS Comput. Biol. 12, 1-21 (2016).

46. Meylan, S. et al. Carbon sources tune antibiotic susceptibility in Pseudomonas aeruginosa via tricarboxylic acid cycle control. Cell Chem. Biol. 24, 1-12 (2017).

47. Zalis, E. A. et al. Stochastic variation in expression of the tricarboxylic acid cycle produces persister cells. mBio 10, 1-10 (2019).

48. Van den Bergh, B., Michiels, J. E. \& Michiels, J. In Bacterial Persistence: Methods and Protocols (eds Michiels, J. \& Fauvart, M.) Vol. 1333, 131-143 (Springer, 2016).

49. Friedrich, T., Hellwig, P. \& Einsle, O. in A Structural Perspective on Respiratory Complex I: Structure and Function of NADH:Ubiquinone Oxidoreductase (ed. Sazanov, L.) 46, 23-60 (Springer, 2012)..

50. Gnandt, E., Dörner, K., Strampraad, M. F. J., de Vries, S. \& Friedrich, T. The multitude of iron-sulfur clusters in respiratory complex I. Biochim. Biophys. Acta-Bioenerg. 1857, 1068-1072 (2016).

51. Baradaran, R., Berrisford, J. M., Minhas, G. S. \& Sazanov, L. A. Crystal structure of the entire respiratory complex I. Nature 494, 25-30 (2013)

52. Efremov, R. G. \& Sazanov, L. A. Structure of the membrane domain of respiratory complex I. Nature 476, 414-420 (2011).

53. Di Luca, A. et al. Symmetry-related proton transfer pathways in respiratory complex I. Proc. Natl. Acad. Sci. USA 114, E6314-E6321 (2017).

54. Friedrich, T., Dekovic, D. K. \& Burschel, S. Assembly of the Escherichia coli NADH:ubiquinone oxidoreductase (respiratory complex I). Biochim. Biophys. Acta-Bioenerg. 1857, 214-223 (2016).

55. Pohl, T., Uhlmann, M., Kaufenstein, M. \& Friedrich, T. Lambda Red-mediated mutagenesis and efficient large scale affinity purification of the Escherichia coli NADH:ubiquinone oxidoreductase (complex I). Biochemistry 46, 10694-10702 (2007). 
56. Steimle, S. et al. Role of subunit NuoL for proton translocation by respiratory complex I. Biochemistry 50, 3386-3393 (2011).

57. Hoeser, J., Gnandt, E. \& Friedrich, T. Low cost, microcontroller based heating device for multi-wavelength differential scanning fluorimetry. Sci. Rep. 8, 1-10 (2018).

58. Kaila, V. R. I. \& Wikström, M. Architecture of bacterial respiratory chains. Nat. Rev. Microbiol. 19, 319-330 (2021).

59. Allison, K. R., Brynildsen, M. P. \& Collins, J. J. Metabolite-enabled eradication of bacterial persisters by aminoglycosides. Nature 473, 216-220 (2011).

60. Krulwich, T. A., Sachs, G. \& Padan, E. Molecular aspects of bacterial $\mathrm{pH}$ sensing and homeostasis. Nat. Rev. Microbiol. 9, 330-343 (2011).

61. Vesselinova, N., Alexandrov, B. S. \& Wall, M. E. Dynamical model of drug accumulation in bacteria: Sensitivity analysis and experimentally testable predictions. PLoS ONE 11, 1-20 (2016).

62. Cramariuc, O. et al. Mechanism for translocation of fluoroquinolones across lipid membranes. Biochim. Biophys. Acta-Biomembr. 1818, 2563-2571 (2012).

63. Aldred, K. J., Kerns, R. J. \& Osheroff, N. Mechanism of quinolone action and resistance. Biochemistry 53, 1565-1574 (2014).

64. Berg, J., Hung, Y. P. \& Yellen, G. A genetically encoded fluorescent reporter of ATP:ADP ratio. Nat. Methods 6, 161-166 (2009).

65. Martinez, K. A. et al. Cytoplasmic pH response to acid stress in individual cells of Escherichia coli and Bacillus subtilis observed by fluorescence ratio imaging microscopy. Appl. Environ. Microbiol. 78, 3706-3714 (2012).

66. Bearson, S. M. D. et al. Acid shock induction of RpoS is mediated by the mouse virulence gene. Microbiology 178, 2572-2579 (1996).

67. Bak, G., Han, K., Kim, D. \& Lee, Y. Roles of $r$ poS-activating small RNAs in pathways leading to acid resistance of Escherichia coli. Microbiologyopen 3, 15-28 (2014)

68. Battesti, A., Majdalani, N. \& Gottesman, S. The RpoS-mediated general stress response in Escherichia coli. Annu. Rev. Microbiol. 65, 189-213 (2011),

69. Schmidt, A. et al. The quantitative and condition-dependent Escherichia coli proteome. Nat. Biotechnol. 34, 104-110 (2016).

70. Kwan, B. W. et al. Arrested protein synthesis increases persister-like cell formation. Antimicrob. Agents Chemother. 57, 1468-1473 (2013).

71. Tannock, I. F. \& Rotin, D. Acid pH in tumors and its potential for therapeutic exploitation. Cancer Res. 49, 4373-4384 (1989).

72. Urbach, V., Hélix, N., Renaudon, B. \& Harvey, B. J. Cellular mechanisms for apical ATP effects on intracellular $\mathrm{pH}$ in human bronchial epithelium. J. Physiol. 543, 13-21 (2002).

73. Kanaporis, G., Treinys, R., Fischmeister, R. \& Jurevičius, J. Metabolic inhibition reduces cardiac L-type $\mathrm{Ca}^{2+}$ channel current due to acidification caused by ATP hydrolysis. PLOS ONE 12, 1-17 (2017).

74. Vroman, R. et al. Extracellular ATP hydrolysis inhibits synaptic transmission by increasing $\mathrm{pH}$ buffering in the synaptic cleft. PLoS Biol. 12, e1001864 (2014).

75. Munder, M. C. et al. A pH-driven transition of the cytoplasm from a fluid- to a solid-like state promotes entry into dormancy. Elife 5, 1-30 (2016).

76. Bruni, G. N. \& Kralj, J. M. Membrane voltage dysregulation driven by metabolic dysfunction underlies bactericidal activity of aminoglycosides. Elife 9, 1-25 (2020).

77. Voskuil, M. I., Covey, C. R. \& Walter, N. D. in Advances in Microbial Physiology Vol. 73, 77-122 (Elsevier Ltd., 2018).

78. Lee, D. D. et al. Magnesium flux modulates ribosomes to increase bacterial survival. Cell 177, 352-360.e13 (2019).

79. Zambrano, M. M. \& Kolter, R. Escherichia coli mutants lacking NADH dehydrogenase I have a competitive disadvantage in stationary phase. $J$. Bacteriol. 175, 5642-5647 (1993).

80. Shan, Y., Lazinski, D. W., Rowe, S. E., Camilli, A. \& Lewis, K. Genetic basis of persister tolerance to aminoglycosides in Escherichia coli. mBio 6, e00078-15 (2015).

81. Girgis, H. S. H. H. S., Hottes, A. K. A. \& Tavazoie, S. Genetic architecture of intrinsic antibiotic susceptibility. PLoS ONE 4, e5629 (2009).

82. Jahn, L. J., Munck, C., Ellabaan, M. M. H. \& Sommer, M. O. A. Adaptive laboratory evolution of antibiotic resistance using different selection regimes lead to similar phenotypes and genotypes. Front. Microbiol. 8, 1-14 (2017).

83. Lázár, V. et al. Bacterial evolution of antibiotic hypersensitivity. Mol. Syst. Biol. 9, 700 (2013).

84. Schurek, K. N. et al. Novel genetic determinants of low-level aminoglycoside resistance in Pseudomonas aeruginosa. Antimicrob. Agents Chemother. 52, 4213-4219 (2008).

85. Ezraty, B. et al. Fe-S cluster biosynthesis controls uptake of aminoglycosides in a ROS-less death pathway. Science 340, 1583-1587 (2013).

86. Wong, K. S. et al. The MoxR ATPase RavA and its cofactor ViaA interact with the NADH:ubiquinone oxidoreductase I in Escherichia coli. PLoS One 9, e85529 (2014).

87. McCollister, B. D., Hoffman, M., Husain, M. \& Vázquez-Torres, A. Nitric oxide protects bacteria from aminoglycosides by blocking the energy- dependent phases of drug uptake. Antimicrob. Agents Chemother. 55, 2189-2196 (2011).

88. Chareyre, S., Barras, F. \& Mandin, P. A small RNA controls bacterial sensitivity to gentamicin during iron starvation. PLOS Genet. 15, e1008078 (2019).

89. Santi, I., Manfredi, P., Maffei, E., Egli, A. \& Jenal, U. Evolution of antibiotic tolerance shapes resistance development in chronic Peudomonas aeruginosa infections. mBio 12, 1-17 (2021).

90. Mühlbauer, M. E. et al. Water-gated proton transfer dynamics in respiratory complex I. J. Am. Chem. Soc. 142, 13718-13728 (2020).

91. Imamovic, L. \& Sommer, M. O. A. Use of collateral sensitivity networks to design drug cycling protocols that avoid resistance development. Sci. Transl. Med. 5, 204ra132 (2013).

92. Chakraborty, S., Mizusaki, H. \& Kenney, L. J. A FRET-based DNA biosensor tracks OmpR-dependent acidification of Salmonella during macrophage infection. PLOS Biol. 13, e1002116 (2015).

93. Helaine, S. et al. Internalization of Salmonella by macrophages induces formation of nonreplicating persisters. Science 343, 204-208 (2014).

94. Vega, N. M., Allison, K. R., Khalil, A. S. \& Collins, J. J. Signaling-mediated bacterial persister formation. Nat. Chem. Biol. 8, 431-433 (2012).

95. Vega, N. M., Allison, K. R., Samuels, A. N., Klempner, M. S. \& Collins, J. J. Salmonella Typhimurium intercepts Escherichia coli signaling to enhance antibiotic tolerance. Proc. Natl. Acad. Sci. USA 110, 14420-14425 (2013).

96. Kuczyńska-Wiśnik, D. et al. Lack of intracellular trehalose affects formation of Escherichia coli persister cells. Microbiology 161, 786-796 (2014).

97. Zarkan, A. et al. Indole pulse signalling regulates the cytoplasmic $\mathrm{pH}$ of $E$. coli in a memory-like manner. Sci. Rep. 9, 1-10 (2019).

98. Goode, O. et al. Persister Escherichia coli cells have a lower intracellular $\mathrm{pH}$ than susceptible cells but maintain their $\mathrm{pH}$ in response to antibiotic treatment. mBio 12, e0090921 (2021).

99. Bartek, I. L. et al. Antibiotic bactericidal activity is countered by maintaining $\mathrm{pH}$ homeostasis in Mycobacterium smegmatis. mSphere 1, 1-18 (2016)

100. Reyes-Fernández, E. Z. \& Schuldiner, S. Acidification of cytoplasm in Escherichia coli provides a strategy to cope with stress and facilitates development of antibiotic resistance. Sci. Rep. 10, 1-13 (2020).

101. Swerdlow, B. M., Setlow, B. \& Setlow, P. Levels of $\mathrm{H}^{+}$and other monovalent cations in dormant and germinating spores of Bacillus megaterium. J. Bacteriol. 148, 20-29 (1981)

102. Setlow, B. \& Setlow, P. Measurements of the $\mathrm{pH}$ within dormant and germinated bacterial spores. Proc. Natl. Acad. Sci. USA 77, 2474-2476 (1980).

103. Magill, N. G., Cowan, A. E., Koppel, D. E. \& Setlow, P. The internal pH of the forespore compartment of Bacillus megaterium decreases by about $1 \mathrm{pH}$ unit during sporulation. J. Bacteriol. 176, 2252-2258 (1994).

104. Dechant, R. C. et al. Cytosolic pH is a second messenger for glucose and regulates the PKA pathway through V-ATPase. EMBO J. 29, 2515-2526 (2010).

105. Robert, F., Gendraud, M. \& Pétel, G. Using intracellular pH to evaluate growth inhibition of strawberry plants. Plant Physiol. Biochem. 37, 155-160 (1999).

106. Busa, W. \& Crowe, J. Intracellular $\mathrm{pH}$ regulates transitions between dormancy and development of brine shrimp (Artemia salina) embryos. Science 221, 366-368 (1983).

107. Bergkessel, M. Regulation of protein biosynthetic activity during growth arrest. Curr. Opin. Microbiol. 57, 62-69 (2020).

108. Dijksterhuis, J., Nijsse, J., Hoekstra, F. A. \& Golovina, E. A. High viscosity and anisotropy characterize the cytoplasm of fungal dormant stress-resistant spores. Eukaryot. Cell 6, 157-170 (2007).

109. Cowan, A. E., Koppel, D. E., Setlow, B. \& Setlow, P. A soluble protein is immobile in dormant spores of Bacillus subtilis but is mobile in germinated spores: Implications for spore dormancy. Proc. Natl. Acad. Sci. USA 100, 4209-4214 (2003).

110. Ablett, S., Darke, A. H., Lillford, P. J. \& Martin, D. R. Glass formation and dormancy in bacterial spores. Int. J. Food Sci. Technol. 34, 59-69 (1999).

111. Parry, B. R. et al. The bacterial cytoplasm has glass-like properties and is fluidized by metabolic activity. Cell 156, 183-194 (2014).

112. Leszczyńska, D. et al. The formation of persister cells in stationary-phase cultures of Escherichia coli is associated with the aggregation of endogenous proteins. PLoS ONE 8, e54737 (2013).

113. Yu, J., Liu, Y., Yin, H. \& Chang, Z. Regrowth-delay body as a bacterial subcellular structure marking multidrug-tolerant persisters. Cell Discov. 5, 8 (2019).

114. Goode, O. et al. Heterologous protein expression favors the formation of protein aggregates in persister and viable but nonculturable bacteria. ACS Infect. Dis. (2021). https://doi.org/10.1021/acsinfecdis.1c00154 (2021).

115. Dewachter, L. et al. The dynamic transition of persistence towards the VBNC state during stationary phase is driven by protein aggregation. mBio 12, e0070321 (2021).

116. Khare, A. \& Tavazoie, S. Extreme antibiotic persistence via heterogeneitygenerating mutations targeting translation. mSystems 5, 1-12 (2020). 
117. Rozov, A. et al. Importance of potassium ions for ribosome structure and function revealed by long-wavelength X-ray diffraction. Nat. Commun. 10, 1-12 (2019).

118. Kaldalu, N., Hauryliuk, V. \& Tenson, T. Persisters-as elusive as ever. Appl. Microbiol. Biotechnol. 100, 6545-6553 (2016).

119. Amato, S. M. et al. The role of metabolism in bacterial persistence. Front. Microbiol. 5, 70 (2014)

120. Wu, Y., Vulić, M., Keren, I. \& Lewis, K. Role of oxidative stress in persister tolerance. Antimicrob. Agents Chemother. 56, 4922-4926 (2012).

121. Thierauf, A., Perez, G. \& Maloy, A. S. Generalized transduction. Methods Mol. Biol. 501, 267-286 (2009).

122. Datsenko, K. A. \& Wanner, B. L. One-step inactivation of chromosomal genes in Escherichia coli K-12 using PCR products. Proc Natl Acad Sci USA 97, 6640-6645 (2000).

123. Baba, T. et al. Construction of Escherichia coli K-12 in-frame, singlegene knockout mutants: the Keio collection. Mol. Syst. Biol. 2, 2006.0008 (2006).

124. Good, N. E. et al. Hydrogen ion buffers for biological research. Biochemistry 5 , 467-477 (1966).

125. Wiegand, I., Hilpert, K. \& Hancock, R. E. W. Agar and broth dilution methods to determine the minimal inhibitory concentration (MIC) of antimicrobial substances. Nat. Protoc. 3, 163-175 (2008).

126. Liebens, V. et al. Identification and characterization of an anti-pseudomonal dichlorocarbazol derivative displaying anti-biofilm activity. Bioorg. Med. Chem. Lett. 24, 5404-5408 (2014).

127. Vedelaar, S., Radzikowski, J. L. \& Heinemann, M. A robust method for generating, quantifying and testing large amounts of Escherichia coli persisters. (2020). https://doi.org/10.1101/2020.07.06.181891 (2020).

128. Omasits, U., Ahrens, C. H., Müller, S. \& Wollscheid, B. Protter: Interactive protein feature visualization and integration with experimental proteomic data. Bioinformatics 30, 884-886 (2014).

129. Zhao, G. \& London, E. An amino acid "transmembrane tendency" scale that approaches the theoretical limit to accuracy for prediction of transmembrane helices: Relationship to biological hydrophobicity. Protein Sci. 15, 1987-2001 (2006).

130. Waterhouse, A. et al. SWISS-MODEL: Homology modelling of protein structures and complexes. Nucleic Acids Res. 46, W296-W303 (2018)

131. Russell, R. B. \& Barton, G. J. Multiple protein sequence alignment from tertiary structure comparison: assignment of global and residue confidence levels. Proteins Struct. Funct. Bioinform. 14, 309-323 (1992).

132. Humphrey, W., Dalke, A. \& Schulten, K. VMD-visual molecular dynamics. J. Mol. Graph. 14, 33-38 (1996).

133. Schrödinger, L. The PyMOL molecular graphics system, Version 2.0. (2015).

134. Gornall, A. G., Bardawill, C. J. \& David, M. M. Determination of serum proteins of the biuret reaction. J. Biol. Chem. 177, 751-766 (1949).

135. Schagger, H., Cramer, W. A. \& Vonjagow, G. Analysis of molecular masses and oligomeric states of protein complexes by blue native electrophoresis and isolation of membrane protein complexes by two-dimensional native electrophoresis. Anal. Biochem. 217, 220-230 (1994).

136. Forneris, F., Orru, R., Bonivento, D., Chiarelli, L. R. \& Mattevi, A. ThermoFAD, a Thermofluor-adapted flavin ad hoc detection system for protein folding and ligand binding. FEBS J. 276, 2833-2840 (2009).

137. Alexandrov, A. I., Mileni, M., Chien, E. Y. T., Hanson, M. A. \& Stevens, R. C. Microscale fluorescent thermal stability assay for membrane proteins. Structure 16, 351-359 (2008).

138. Stolpe, S. \& Friedrich, T. The Escherichia coli NADH:ubiquinone oxidoreductase (complex I) is a primary proton pump but may be capable of secondary sodium antiport. J. Biol. Chem. 279, 18377-18383 (2004).

139. Kaback, H. R. Transport studies in bacterial membrane vesicles. Science $\mathbf{1 8 6}$ 882-892 (1974).

140. Tafin, U. F. et al. Gentamicin improves the activities of daptomycin and vancomycin against Enterococcus faecalis in vitro and in an experimental foreign-body infection model. Antimicrob. Agents Chemother. 55, 4821-4827 (2011).

141. Miesenböck, G., Angelis, D. D. E. \& Rothman, J. Visualizing secretion and synaptic transmission with $\mathrm{pH}$-sensitive green fluorescent proteins. Nature 394, 192-195 (1998)

142. Glatter, T. et al. Large-scale quantitative assessment of different in-solution protein digestion protocols reveals superior cleavage efficiency of tandem LysC/trypsin proteolysis over trypsin digestion. J. Proteome Res. 11, 5145-5156 (2012).

143. Kanjee, U. et al. Linkage between the bacterial acid stress and stringent responses: the structure of the inducible lysine decarboxylase. EMBO J 30, 931-944 (2011).

144. Bougdour, A. \& Gottesman, S. ppGpp regulation of RpoS degradation via anti-adaptor protein IraP. Proc. Natl. Acad. Sci. USA 104, 12896-12901 (2007).
145. Lee, J.-W., Park, Y.-H. \& Seok, Y.-J. Rsd balances (p)ppGpp level by stimulating the hydrolase activity of SpoT during carbon source downshift in Escherichia coli. Proc. Natl. Acad. Sci. USA 115, E6845-E6854 (2018).

146. Hauryliuk, V., Atkinson, G. C., Murakami, K. S., Tenson, T. \& Gerdes, K. Recent functional insights into the role of (p)ppGpp in bacterial physiology. Nat. Rev. Microbiol. 13, 298-309 (2015).

147. Bradford, M. M. A rapid and sensitive method for the quantitation of microgram quantities of protein utilizing the principle of protein-dye binding. Anal. Biochem. 72, 248-254 (1976).

148. Sutter, M. et al. Structural basis of enzyme encapsulation into a bacterial nanocompartment. Nat. Struct. Mol. Biol. 15, 939-947 (2008).

149. Contreras, H. et al. Characterization of a Mycobacterium tuberculosis nanocompartment and its potential cargo proteins. J. Biol. Chem. 289, 18279-18289 (2014).

150. Lončar, N., Rozeboom, H. J., Franken, L. E., Stuart, M. C. A. \& Fraaije, M. W Structure of a robust bacterial protein cage and its application as a versatile biocatalytic platform through enzyme encapsulation. Biochem. Biophys. Res. Commun. 529, 548-553 (2020).

151. Tamura, A. et al. Packaging guest proteins into the encapsulin nanocompartment from Rhodococcus erythropolis N771. Biotechnol. Bioeng. 112, 13-20 (2015)

152. Heinemeyer, E.-A. \& Richter, D. In vitro degradation of guanosine tetraphosphate (ppGpp) by an enzyme associated with the ribosomal fraction from Escherichia coli. FEBS Lett. 84, 357-361 (1977).

153. An, G., Justesen, J., Watson, R. J. \& Friesen, J. D. Cloning the spoT gene of Escherichia coli: Identification of the spoT gene product. J. Bacteriol. 137, 1100-1110 (1979).

154. Gnandt, E. \& Friedrich, T. The Mechanism of Nadh Oxidation by Respiratory Complex I With Focus on the Production of Reactive Oxygen Species (Universität, 2018).

\section{Acknowledgements}

The authors thank Susan Gottesman for the AB037 strain, Joan L. Slonczewski for the pHluorin plasmid, Steven B. Vik for the BA14 strain and Henri de Greve for the UTI89 strain. We further thank Ying Liu for help with cloning the plasmid for encapsulin-SpoT expression, Franziska Number for providing controls for the ACMA quenching, Alexandros Papagiannakis for valuable suggestions as well as for help with microscopy and Bert Poolman for valuable discussions. B.V.D.B. is recipient of fellowships from the Fund for Scientific Research, Flanders (FWO; 12O1917N, 12O1922N, V428917N, and 11C6812/4N), from the Federation of European Microbiological Societies (FEMS; RG-2016-0052), from the Belgian American Educational Foundation (BAEF 2016-E083) and from the European Molecular Biology Organization (EMBO; ALTF 344 2017). J.E.M. was granted a fellowship by the Agency for Innovation by Science and Technology (IWT). This work was furthermore supported by grants from FWO (1528318N, G0B2515N and G055517N), KU Leuven (C16/17/006), the Flanders Institute for Biotechnology (VIB) (to J.M. and B.V.D.B), the Deutsche Forschungsgemeinschaft (278002225/RTG 2202) (to T.F.) and from the Dutch Research Council (NWO; VIDI grant 864.11.001) to M.H.

\section{Author contributions}

Conceptualization, B.V.D.B., H.S., J.L.R., M.F., T.F., J.M. and M.H.; Methodology, B.V.D.B., H.S., J.E.M., J.L.R., A. S., M.F., T.F., J.M. and M.H.; Investigation, B.V.D.B. H.S., J.E.M., T.E.P.K., J.L.R., T.M., N.L., J.S., S.V., L.D., A.S.; Writing-Original Draft, B.V.D.B., H.S., J.L.R., J.M. and M.H.; Writing-Review \& Editing, B.V.D.B., H.S., J.M. and M.H.; Funding Acquisition, B.V.D.B., T.F., J.M. and M.H.; Resources, A.S.,T.F., J.M., and M.H.; Supervision, J.M. and M.H.

\section{Competing interests}

The authors declare no competing interests.

\section{Additional information}

Supplementary information The online version contains supplementary material available at https://doi.org/10.1038/s41467-022-28141-x.

Correspondence and requests for materials should be addressed to Jan Michiels or Matthias Heinemann.

Peer review information Nature Communications thanks Gregory Cook and the other, anonymous, reviewers for their contribution to the peer review of this work. Peer reviewer reports are available.

Reprints and permission information is available at http://www.nature.com/reprints

Publisher's note Springer Nature remains neutral with regard to jurisdictional claims in published maps and institutional affiliations. 
(c) (i) Open Access This article is licensed under a Creative Commons Attribution 4.0 International License, which permits use, sharing, adaptation, distribution and reproduction in any medium or format, as long as you give appropriate credit to the original author(s) and the source, provide a link to the Creative Commons license, and indicate if changes were made. The images or other third party material in this article are included in the article's Creative Commons license, unless indicated otherwise in a credit line to the material. If material is not included in the article's Creative Commons license and your intended use is not permitted by statutory regulation or exceeds the permitted use, you will need to obtain permission directly from the copyright holder. To view a copy of this license, visit http://creativecommons.org/ licenses/by/4.0/.

(C) The Author(s) 2022 